\title{
Euclid: Forecasts from redshift-space distortions and the Alcock-Paczynski test with cosmic voids ${ }^{\star}$
}

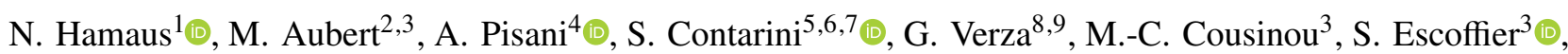
A. Hawken ${ }^{3}$, G. Lavaux ${ }^{10}$, G. Pollina ${ }^{1}$, B. D. Wandelt ${ }^{10} \odot$, J. Weller ${ }^{1,11}$, M. Bonici ${ }^{12,13}$, C. Carbone ${ }^{14,15}$, L. Guzzo ${ }^{14,16,17}$, A. Kovacs ${ }^{18,19}$, F. Marulli6,20,21, E. Massara ${ }^{22,23}$, L. Moscardini $6,20,21$, P. Ntelis ${ }^{3}$,

W. J. Percival ${ }^{22,23,24}$, S. Radinović ${ }^{25}$, M. Sahlén ${ }^{26,27}$, Z. Sakr ${ }^{28,29}$, A. G. Sánchez ${ }^{11}$, H. A. Winther ${ }^{25}$, N. Auricchio ${ }^{21}$, S. Awan $^{30}$, R. Bender ${ }^{1,11}$, C. Bodendorf ${ }^{11}$, D. Bonino ${ }^{31}$, E. Branchini ${ }^{32,33}$, M. Brescia ${ }^{34}$, J. Brinchmann $^{35}$, V. Capobianco ${ }^{31}$, J. Carretero ${ }^{36,37}$, F. J. Castander ${ }^{38,39}$, M. Castellano ${ }^{40}$, S. Cavuoti ${ }^{34,41,42}$, A. Cimatti ${ }^{5,43}$, R. Cledassou ${ }^{44,45}$, G. Congedo ${ }^{46}$, L. Conversi ${ }^{47,48}$, Y. Copin $^{49}$, L. Corcione ${ }^{31}$, M. Cropper ${ }^{30}$, A. Da Silva ${ }^{50,51}$, H. Degaudenzi ${ }^{52}$, M. Douspis ${ }^{53}$, F. Dubath ${ }^{52}$, C. A. J. Duncan ${ }^{54}$, X. Dupac ${ }^{48}$, S. Dusini ${ }^{8}$, A. Ealet ${ }^{49}$, S. Ferriol ${ }^{49}$, P. Fosalba ${ }^{38,39}$, M. Frailis ${ }^{55}$, E. Franceschi ${ }^{21}$, P. Franzetti ${ }^{15}$, M. Fumana ${ }^{15}$, B. Garilli ${ }^{15}$, B. Gillis ${ }^{46}$, C. Giocoli ${ }^{56,57}$, A. Grazian ${ }^{58}$, F. Grupp ${ }^{1,11}$, S. V. H. Haugan ${ }^{25}$, W. Holmes ${ }^{59}$, F. Hormuth ${ }^{60,61}$, K. Jahnke ${ }^{61}$, S. Kermiche ${ }^{3}$, A. Kiessling ${ }^{59}$, M. Kilbinger ${ }^{62}$, T. Kitching ${ }^{30}$, M. Kümmel ${ }^{1}$, M. Kunz ${ }^{63}$, H. Kurki-Suonio ${ }^{64}$, S. Ligori ${ }^{31}$, P. B. Lilje ${ }^{25}$, I. Lloro ${ }^{65}$, E. Maiorano ${ }^{21}$, O. Marggraf ${ }^{66}$, K. Markovic ${ }^{59}$, R. Massey ${ }^{67}$, S. Maurogordato ${ }^{68}$, M. Melchior ${ }^{69}$, M. Meneghetti ${ }^{6,21,70}$, G. Meylan ${ }^{71}$, M. Moresco ${ }^{20,21}$, E. Munari ${ }^{55}$, S. M. Niemi ${ }^{72}$, C. Padilla ${ }^{37}$, S. Paltani ${ }^{52}$, F. Pasian ${ }^{55}$, K. Pedersen ${ }^{73}$, V. Pettorino ${ }^{62}$, S. Pires ${ }^{62}$, M. Poncet ${ }^{45}$, L. Popa ${ }^{74}$, L. Pozzetti $^{21}$, R. Rebolo ${ }^{19,75}$, J. Rhodes ${ }^{59}$, H. Rix ${ }^{61}$, M. Roncarelli ${ }^{20,21}$, E. Rossetti ${ }^{20}$, R. Saglia ${ }^{1,11}$, P. Schneider ${ }^{66}$, A. Secroun ${ }^{3}$, G. Seidel ${ }^{61}$, S. Serrano ${ }^{38,39}$, C. Sirignano ${ }^{8,9}$, G. Sirri6 ${ }^{\text {, J.-L. Starck }}{ }^{62}$, P. Tallada-Crespí ${ }^{36,76}$, D. Tavagnacco ${ }^{55}$, A. N. Taylor ${ }^{46}$, I. Tereno ${ }^{50,77}$, R. Toledo-Moreo ${ }^{78}$, F. Torradeflot ${ }^{36,76}$, E. A. Valentijn ${ }^{79}$, L. Valenziano ${ }^{6,21}$, Y. Wang ${ }^{80}$, N. Welikala ${ }^{46}$, G. Zamorani ${ }^{21}$, J. Zoubian ${ }^{3}$, S. Andreon ${ }^{17}$, M. Baldi ${ }^{6,21,81}$, S. Camera ${ }^{31,82,83}$, S. Mei ${ }^{84}$, C. Neissner ${ }^{36,37}$, and E. Romelli ${ }^{55}$

(Affiliations can be found after the references)

Received 23 August 2021 / Accepted 31 October 2021

\section{ABSTRACT}

Euclid is poised to survey galaxies across a cosmological volume of unprecedented size, providing observations of more than a billion objects distributed over a third of the full sky. Approximately 20 million of these galaxies will have their spectroscopy available, allowing us to map the three-dimensional large-scale structure of the Universe in great detail. This paper investigates prospects for the detection of cosmic voids therein and the unique benefit they provide for cosmological studies. In particular, we study the imprints of dynamic (redshift-space) and geometric (Alcock-Paczynski) distortions of average void shapes and their constraining power on the growth of structure and cosmological distance ratios. To this end, we made use of the Flagship mock catalog, a state-of-the-art simulation of the data expected to be observed with Euclid. We arranged the data into four adjacent redshift bins, each of which contains about 11000 voids and we estimated the stacked void-galaxy cross-correlation function in every bin. Fitting a linear-theory model to the data, we obtained constraints on $f / b$ and $D_{\mathrm{M}} H$, where $f$ is the linear growth rate of density fluctuations, $b$ the galaxy bias, $D_{\mathrm{M}}$ the comoving angular diameter distance, and $H$ the Hubble rate. In addition, we marginalized over two nuisance parameters included in our model to account for unknown systematic effects in the analysis. With this approach, Euclid will be able to reach a relative precision of about $4 \%$ on measurements of $f / b$ and $0.5 \%$ on $D_{\mathrm{M}} H$ in each redshift bin. Better modeling or calibration of the nuisance parameters may further increase this precision to $1 \%$ and $0.4 \%$, respectively. Our results show that the exploitation of cosmic voids in Euclid will provide competitive constraints on cosmology even as a stand-alone probe. For example, the equation-of-state parameter, $w$, for dark energy will be measured with a precision of about $10 \%$, consistent with previous more approximate forecasts.

Key words. cosmology: observations - cosmological parameters - dark energy - large-scale structure of Universe - methods: data analysis surveys

\section{Introduction}

The formation of cosmic voids in the large-scale structure of the Universe is a consequence of the gravitational interaction of its initially smooth distribution of matter eventually evolving into collapsed structures that make up the cosmic web (Zeldovich 1970). This process leaves behind vast regions of

^ This paper is published on behalf of the Euclid Consortium. nearly empty space that constitute the largest known structures in the Universe. Since their first discovery in the late 1970 s (Gregory \& Thompson 1978; Jõeveer et al. 1978), cosmic voids have intrigued scientists given their peculiar nature (e.g., Kirshner et al. 1981; Bertschinger 1985; White et al. 1987; van de Weygaert \& van Kampen 1993; Peebles 2001). However, only the recent advances in surveys, such as 6dFGS (Jones et al. 2004), BOSS (Dawson et al. 2013), DES (The Dark Energy Survey Collaboration 2005), eBOSS (Dawson et al. 2016), 
KiDS (de Jong et al. 2013), SDSS (Eisenstein et al. 2011), and VIPERS (Guzzo et al. 2014), have enabled systematic studies of statistically significant sample sizes (e.g., Pan et al. 2012; Sutter et al. 2012a), placing the long-overlooked field of cosmic voids into a new focus of interest in astronomy. In conjunction with the extensive development of large simulations (e.g., Springel 2005; Schaye et al. 2015; Dolag et al. 2016; Potter et al. 2017), this has sparked a plethora of studies on voids and their connection to galaxy formation (Hoyle et al. 2005; Patiri et al. 2006; Kreckel et al. 2012; Ricciardelli et al. 2014; Habouzit et al. 2020; Panchal et al. 2020), large-scale structure (Sheth \& van de Weygaert 2004; Hahn et al. 2007; van de Weygaert \& Schaap 2009; Jennings et al. 2013; Hamaus et al. 2014a; Chan et al. 2014; Voivodic et al. 2020), the nature of gravity (Clampitt et al. 2013; Zivick et al. 2015; Cai et al. 2015; Barreira et al. 2015; Achitouv 2016; Voivodic et al. 2017; Falck et al. 2018; Sahlén \& Silk 2018; Baker et al. 2018; Paillas et al. 2019; Davies et al. 2019; Perico et al. 2019; Alam et al. 2021; Contarini et al. 2021; Wilson \& Bean 2021), properties of dark matter (Leclercq et al. 2015; Yang et al. 2015; Reed et al. 2015; Baldi \& VillaescusaNavarro 2018), dark energy (Lee \& Park 2009; Bos et al. 2012; Spolyar et al. 2013; Pisani et al. 2015a; Pollina et al. 2016; Verza et al. 2019), massive neutrinos (Massara et al. 2015; Banerjee \& Dalal 2016; Sahlén 2019; Kreisch et al. 2019; Schuster et al. 2019; Zhang et al. 2020; Bayer et al. 2021), inflation (Chan et al. 2019), and cosmology in general (Lavaux \& Wandelt 2012; Sutter et al. 2012b; Hamaus et al. 2014b, 2016, 2020; Correa et al. 2019, 2022; Contarini et al. 2019; Nadathur et al. 2019, 2020; Paillas et al. 2021; Kreisch et al. 2021). We refer to Pisani et al. (2019) for a more extensive recent summary.

From an observational perspective, voids are an abundant structure type that, together with halos, filaments, and walls, build up the cosmic web. It is therefore natural to utilize them in the search for those observables that have traditionally been measured via galaxies or galaxy clusters, which trace the overdense regions of large-scale structure. This strategy has proven itself to be very promising in recent years, uncovering a treasure trove of untapped signals that carry cosmologically relevant information, such as the integrated Sachs-Wolfe (ISW; Granett et al. 2008; Cai et al. 2010, 2014; Ilić et al. 2013; Nadathur \& Crittenden 2016; Kovács \& García-Bellido 2016; Kovács et al. 2019), Sunyaev-Zeldovich (SZ; Alonso et al. 2018), and Alcock-Paczynski (AP) effects (Sutter et al. 2012b, 2014a; Hamaus et al. 2016; Mao et al. 2017; Nadathur et al. 2019), as well as gravitational lensing (Melchior et al. 2014; Clampitt \& Jain 2015; Gruen et al. 2016; Sánchez et al. 2017a; Cai et al. 2017; Chantavat et al. 2017; Brouwer et al. 2018; Fang et al. 2019), baryon acoustic oscillations (BAO; Kitaura et al. 2016; Liang et al. 2016; Chan \& Hamaus 2021; Forero-Sánchez et al. 2021), and redshift-space distortions (RSD; Paz et al. 2013; Cai et al. 2016; Hamaus et al. 2017; Hawken et al. 2017, 2020; Achitouv 2019; Aubert et al. 2020). The aim of this paper is to forecast the constraining power on cosmological parameters with a combined analysis of RSD and the AP effect from voids available in Euclid. As proposed in Hamaus et al. (2015) and carried out with BOSS data in Hamaus et al. (2016) for the first time, this combined approach allows simultaneous constraints on the expansion history of the Universe and the growth rate of structure inside it.

The Euclid satellite mission is a "Stage-IV" dark energy experiment (Albrecht et al. 2006) that will outperform current surveys in the number of observed galaxies and in coverage of cosmological volume (Laureijs et al. 2011). Scheduled for launch in 2022, an assessment of its science performance is timely (Amendola et al. 2018; Euclid Collaboration 2020) and the scientific return that can be expected from voids is being investigated in a series of companion papers within the Euclid Collaboration. These papers cover different observables, such as the void size function (Contarini et al., in prep.), the voidgalaxy cross-correlation function after velocity-field reconstruction (Radinović et al., in prep.), or void lensing (Bonici et al., in prep.), providing independent forecasts on their cosmological constraining power. In this paper, we present a mock-data analysis of the stacked void-galaxy cross-correlation function in redshift space based on the Flagship simulation (Potter et al. 2017), which provides realistic galaxy catalogs as expected to be observed with Euclid. In the following, we outline the theoretical background in Sect. 2, describe the mock data in Sect. 3, and present our results in Sect. 4. The implications of our findings are then discussed in Sect. 5 and our conclusions are summarized in Sect. 6.

\section{Theoretical background}

According to the cosmological principle, the Universe obeys homogeneity and isotropy on very large scales, which is supported by recent observations (e.g., Scrimgeour et al. 2012; Laurent et al. 2016; Ntelis et al. 2017; Gonçalves et al. 2021). However, below the order of $10^{2} \mathrm{Mpc}$ scales, we observe the structures that form the cosmic web, which break these symmetries locally. Nevertheless, the principle is still valid on those scales in a statistical sense, that is, for an ensemble average over patches of similar extent from different locations in the Universe. If the physical size of such patches is known (a so-called standard ruler), this enables an inertial observer to determine cosmological distances and the expansion history. The BAO feature in the galaxy distribution is a famous example for a standard ruler, it has been exploited for distance measurements with great success in the past (e.g., Alam et al. 2017, 2021) and constitutes one of the main cosmological probes of Euclid (Laureijs et al. 2011).

A related approach may be pursued with so-called standard spheres, namely, patches of a known physical shape (in particular, spherically symmetric ones). This method has originally been proposed by Alcock \& Paczynski (1979, hereafter AP) as a probe of the expansion history, it was later demonstrated that voids are well suited for this type of experiment (Ryden 1995; Lavaux \& Wandelt 2012). In principle, it applies to any type of structure in the Universe that exhibits random orientations (such as halos, filaments, or walls), which necessarily results in a spherically symmetric ensemble average. However, the expansion history can only be probed with structures that have not decoupled from the Hubble flow via gravitational collapse. Furthermore, spherical symmetry is broken by peculiar line-of-sight motions of the observed objects that make up this structure. These cause a Doppler shift in the received spectrum and, hence, affect the redshift-distance relation to the source (Kaiser 1987). In order to apply the AP test, one has to account for those RSD, which requires the modeling of peculiar velocities. For the complex phase-space structure of halos, respectively galaxy clusters as their observational counterparts, this is a very challenging problem. For filaments and walls, the situation is only marginally improved, since they have experienced shell crossing in at least one dimension. On the other hand, voids have hardly undergone any shell crossing in their interiors (Shandarin 2011; Abel et al. 2012; Sutter et al. 2014b; Hahn et al. 2015), providing an environment that is characterized by a coherent flow of matter and on that is therefore very amenable to dynamical models. 
In fact, with the help of $N$-body simulations, it has been shown that local mass conservation provides a very accurate description, even at linear order in the density fluctuations (Hamaus et al. 2014c). In that case, the velocity field $\boldsymbol{u}$ relative to the void center is given by (Peebles 1980)

$\boldsymbol{u}(\boldsymbol{r})=-\frac{f(z)}{3} \frac{H(z)}{1+z} \Delta(r) \boldsymbol{r}$,

where $\boldsymbol{r}$ is the comoving real-space distance vector to the void center, $H(z)$ the Hubble rate at redshift $z, f(z)$ is the linear growth rate of density perturbations $\delta$, and $\Delta(r)$ is the average matterdensity contrast enclosed in a spherical region of radius $r$ :

$\Delta(r)=\frac{3}{r^{3}} \int_{0}^{r} \delta\left(r^{\prime}\right) r^{\prime 2} \mathrm{~d} r^{\prime}$

The comoving distance vector $s$ in redshift space receives an additional contribution from the line-of-sight component of $\boldsymbol{u}$ (indicated by $\boldsymbol{u}_{\|}$), caused by the Doppler effect,

$\boldsymbol{s}=\boldsymbol{r}+\frac{1+z}{H(z)} \boldsymbol{u}_{\|}=\boldsymbol{r}-\frac{f(z)}{3} \Delta(r) \boldsymbol{r}_{\|}$.

This equation determines the mapping between real and redshift space at linear order. Its Jacobian can be expressed analytically and yields a relation between the void-galaxy cross-correlation functions $\xi$ in both spaces (a superscript $s$ indicates redshift space),

$\xi^{s}(s) \simeq \xi(r)+\frac{f}{3} \Delta(r)+f \mu^{2}[\delta(r)-\Delta(r)]$,

where $\mu=r_{\|} / r$ denotes the cosine of the angle between $\boldsymbol{r}$ and the line of sight (see Cai et al. 2016; Hamaus et al. 2017, 2020, for a more detailed derivation). The real-space quantities $\xi(r), \delta(r)$ and its integral $\Delta(r)$ on the right-hand side of Eq. (4) are a priori unknown, but they can be related to the observables with some basic assumptions. Firstly, $\xi(r)$ can be obtained via deprojection of the projected void-galaxy cross-correlation function $\xi_{\mathrm{p}}^{s}\left(s_{\perp}\right)$ in redshift space (Pisani et al. 2014; Hawken et al. 2017),

$\xi(r)=-\frac{1}{\pi} \int_{r}^{\infty} \frac{\mathrm{d} \xi_{\mathrm{p}}^{s}\left(s_{\perp}\right)}{\mathrm{d} s_{\perp}}\left(s_{\perp}^{2}-r^{2}\right)^{-1 / 2} \mathrm{~d} s_{\perp}$.

By construction $\xi_{\mathrm{p}}^{s}\left(s_{\perp}\right)$ is insensitive to RSD, since the line-ofsight component $s_{\|}$is integrated out in its definition and the projected separation $s_{\perp}$ on the plane of the sky is identical to its real-space counterpart $r_{\perp}$,

$\xi_{\mathrm{p}}^{s}\left(s_{\perp}\right)=\int \xi^{s}(s) \mathrm{d} s_{\|}=2 \int_{s_{\perp}}^{\infty} r \xi(r)\left(r^{2}-s_{\perp}^{2}\right)^{-1 / 2} \mathrm{~d} r$.

Equations (5) and (6) are also referred to as inverse and forward Abel transform, respectively (Abel et al. 1842; Bracewell 1999).

Secondly, the matter fluctuation $\delta(r)$ around the void center can be related to $\xi(r)$ assuming a bias relation for the galaxies in that region. Based on simulation studies, it has been demonstrated that a linear relation of the form $\xi(r)=b \delta(r)$ with a proportionality constant $b$ serves that purpose with sufficiently high accuracy. Moreover, it has been shown that the value of $b$ is linearly related to the large-scale linear galaxy bias of the tracer distribution, and coincides with it for sufficiently large voids (Sutter et al. 2014c; Pollina et al. 2017, 2019; Contarini et al. 2019; Ronconi et al. 2019). With this, Eq. (4) can be expressed as

$\xi^{s}(s) \simeq \xi(r)+\frac{1}{3} \frac{f}{b} \bar{\xi}(r)+\frac{f}{b} \mu^{2}[\xi(r)-\bar{\xi}(r)]$, where

$\bar{\xi}(r)=3 r^{-3} \int_{0}^{r} \xi\left(r^{\prime}\right) r^{\prime 2} \mathrm{~d} r^{\prime}$

Now, together with Eq. (3) to relate real and redshift-space coordinates, Eq. (7) provides a description of the observable voidgalaxy cross-correlation function at linear order in perturbation theory.

In order to determine the distance vector $\boldsymbol{s}$ for a given voidgalaxy pair, it is necessary to convert their observed separation in angle $\delta \vartheta$ and redshift $\delta z$ to comoving distances via

$s_{\perp}=D_{\mathrm{M}}(z) \delta \vartheta, \quad s_{\|}=\frac{c}{H(z)} \delta z$

where $D_{\mathrm{M}}(z)$ is the comoving angular diameter distance. Both $H(z)$ and $D_{\mathrm{M}}(z)$ depend on cosmology, so any evaluation of Eq. (9) requires the assumption of a fiducial cosmological model. To maintain the full generality of the model, it is customary to introduce two AP parameters that inherit the dependence on cosmology via (e.g., Sánchez et al. 2017b)

$q_{\perp}=\frac{s_{\perp}^{*}}{s_{\perp}}=\frac{D_{\mathrm{M}}^{*}(z)}{D_{\mathrm{M}}(z)}, \quad q_{\|}=\frac{s_{\|}^{*}}{s_{\|}}=\frac{H(z)}{H^{*}(z)}$.

In this notation, the starred quantities are evaluated in the true underlying cosmology, which is unknown, while the un-starred ones correspond to the assumed fiducial values of $D_{\mathrm{M}}$ and $H$. Equation (9) can be rewritten as $s_{\perp}^{*}=q_{\perp} D_{\mathrm{M}}(z) \delta \vartheta$ and $s_{\|}^{*}=$ $q_{\|} c \delta z / H(z)$, which is valid for a wide range of cosmological models. In the special case where the fiducial cosmology coincides with the true one, we have $q_{\perp}=q_{\|}=1$. This method is known as the AP test, providing cosmological constraints via measurements of $D_{\mathrm{M}}(z)$ and $H(z)$. Without an absolute calibration scale the parameters $q_{\perp}$ and $q_{\|}$remain degenerate in the AP test and it is only their ratio,

$\varepsilon=\frac{q_{\perp}}{q_{\|}}=\frac{D_{\mathrm{M}}^{*}(z) H^{*}(z)}{D_{\mathrm{M}}(z) H(z)}$,

that can be determined, providing a measurement of $D_{\mathrm{M}}^{*}(z) H^{*}(z)$. We adopt a flat $\Lambda$ cold dark matter (CDM) cosmology as our fiducial model, where

$D_{\mathrm{M}}(z)=\int_{0}^{z} \frac{c}{H\left(z^{\prime}\right)} \mathrm{d} z^{\prime}, \quad H(z)=H_{0} \sqrt{\Omega_{\mathrm{m}}(1+z)^{3}+\Omega_{\Lambda}}$,

with the present-day Hubble constant, $H_{0}$, matter-density parameter, $\Omega_{\mathrm{m}}$, and cosmological constant parameter, $\Omega_{\Lambda}=1-\Omega_{\mathrm{m}}$. This model includes the true input cosmology of the Flagship simulation with parameter values stated in Sect. 3 below, which is also used for void identification. The impact of the assumed cosmology on the latter has previously been investigated and was found to be negligible (e.g., Hamaus et al. 2020). In Sect. 5, we additionally consider an extended $w C D M$ model to include the equation-of-state parameter, $w$, for dark energy.

\section{Mock catalogs}

\subsection{Flagship simulation}

We employed the Euclid Flagship mock galaxy catalog (version 1.8.4), which is based on an $N$-body simulation of $12600^{3}$ (two trillion) dark matter particles in a periodic box of $3780 \mathrm{~h}^{-1} \mathrm{Mpc}$ on a side (Potter et al. 2017). It adopts a flat $\Lambda \mathrm{CDM}$ cosmology with parameter values $\Omega_{\mathrm{m}}=0.319, \Omega_{\mathrm{b}}=0.049, \Omega_{\Lambda}=0.681$, 
$\sigma_{8}=0.83, n_{\mathrm{s}}=0.96$, and $h=0.67$, as obtained by Planck in 2015 (Planck Collaboration XIII 2016). Dark matter halos are identified with the ROCKSTAR halo finder (Behroozi et al. 2013) and populated with central and satellite galaxies using a halo occupation distribution (HOD) framework to reproduce the relevant observables for Euclid's main cosmological probes. The HOD algorithm (Carretero et al. 2015; Crocce et al. 2015) has been calibrated exploiting several observational constraints, including the local luminosity function for the faintest galaxies (Blanton et al. 2003, 2005) and galaxy clustering statistics as a function of luminosity and color (Zehavi et al. 2011). The simulation box is converted into a light cone that comprises one octant of the sky $\left(5157 \mathrm{deg}^{2}\right)$. The expected footprint of Euclid will cover a significantly larger sky area of roughly $15000 \mathrm{deg}^{2}$ in total.

With its two complementary instruments, the VISible imager (VIS, Cropper et al. 2018) and the Near Infrared Spectrograph and Photometer (NISP, Costille et al. 2018), Euclid will provide photometry and slitless spectroscopy using a near-infrared grism. In this paper, we consider the spectroscopic galaxy sample after a cut in $\mathrm{H} \alpha$ flux, $f_{\mathrm{H} \alpha}>2 \times 10^{-16} \mathrm{erg} \mathrm{s}^{-1} \mathrm{~cm}^{-2}$, which corresponds to the expected detection threshold for Euclid, covering a redshift range of $0.9<z<1.8$ (Laureijs et al. 2011). In addition, we randomly dilute this sample to $60 \%$ of all objects and add a Gaussian redshift error with rms of $\sigma_{z}=0.001$, independent of $z$. This matches the expected median completeness and spectroscopic performance of the survey in a more optimistic scenario and results in a final mock catalog containing about $6.5 \times 10^{6}$ galaxies.

\subsection{VIDE voids}

For the creation of void catalogs we make use of the public Void IDentification and Examination toolkit VIDE ${ }^{1}$ (Sutter et al. 2015). At the core of VIDE is ZOBOV (Neyrinck 2008), which is a watershed algorithm (Platen et al. 2007) that delineates threedimensional basins in the density field of tracer particles. The density field is estimated via Voronoi tessellation, assigning to each tracer particle, $i$, a cell of volume $V_{i}$. In Euclid, these tracer particles are galaxies, distributed over a masked light cone with a redshift-dependent number density, $n_{\mathrm{g}}(z)$. In particular, VIDE provides a framework for handling these complications; we refer to Sutter et al. (2015) for a detailed discussion. Local density minima serve as starting points to identify extended watershed basins whose density monotonically increases among their neighboring cells. All the Voronoi cells that make up such a basin determine a void region that includes its volume-weighted barycenter, which we define as the location of the void center.

Moreover, we assign an effective radius $R$ to every void, which corresponds to a sphere with the same volume:

$R=\left(\frac{3}{4 \pi} \sum_{i} V_{i}\right)^{1 / 3}$

Using VIDE we find a total of $N_{\mathrm{v}}=58601$ voids in the Flagship mock light cone, after discarding those that intersect with the survey mask or redshift boundaries. In addition, we implement a purity selection cut based on the effective radius of a void at redshift $Z$,

$R>N_{\mathrm{s}}\left(\frac{4 \pi}{3} n_{\mathrm{g}}(Z)\right)^{-1 / 3}$.

1 https://bitbucket.org/cosmicvoids/vide_public/
We denote the redshift of void centers with a capital $Z$, to distinguish it from the redshift $z$ of galaxies, while $N_{\mathrm{s}}$ determines the minimum included void size in units of the average tracer separation. The smaller the value of $N_{\mathrm{s}}$, the larger the contamination by spurious voids that may arise via Poisson fluctuations (Neyrinck 2008; Cousinou et al. 2019) and have been misidentified due to RSD (Pisani et al. 2015b; Correa et al. 2021, 2022). We adopt a value of $N_{\mathrm{s}}=3$, which leaves us with a final number of $N_{\mathrm{v}}=44356$ voids with minimum effective radius of $R \simeq$ $18.6 h^{-1} \mathrm{Mpc}$. This sample is further split into 4 consecutive redshift bins with an equal number of voids per bin, $N_{\mathrm{v}}=11089$, see Fig. 1. The selected number of redshift bins is a trade-off between the necessary statistical power to estimate our data vectors and their covariance with sufficient accuracy in each bin, and an adequate sampling of the redshift evolution of $f \sigma_{8}$ and $D_{\mathrm{M}} H$ (see Sect. 4). The removal of voids close to the redshift boundaries of the Flagship light cone causes their abundance to decline, which lowers the statistical constraining power in that regime.

\section{Data analysis}

Our data vector is represented by the void-galaxy crosscorrelation function in redshift space. As this function is anisotropic, we can either consider its $2 \mathrm{D}$ version with coordinates perpendicular to and along the line of sight, $\xi^{s}\left(s_{\perp}, s_{\|}\right)$, or its decomposition into multipoles by use of Legendre polynomials $P_{\ell}$ of order $\ell$,

$\xi_{\ell}^{s}(s)=\frac{2 \ell+1}{2} \int_{-1}^{1} \xi^{s}\left(s, \mu_{s}\right) P_{\ell}\left(\mu_{s}\right) \mathrm{d} \mu_{s}$,

where $\mu_{s}=s_{\|} / s$. We highlight that the notations $\xi^{s}(s), \xi^{s}\left(s_{\perp}, s_{\|}\right)$, and $\xi^{s}\left(s, \mu_{s}\right)$ all refer to the same physical quantity, albeit via their different mathematical formulations. Here, we make use of the full 2D correlation function for our model fits, since it contains all the available information on RSD and AP distortions. For the sake of completeness, we additionally provide the three multipoles of the lowest even order, $\ell=0,2,4$ (i.e., monopole, quadrupole, and hexadecapole). Their theoretical linear predictions directly follow from Eq. (7):

$\xi_{0}^{s}(s)=\left(1+\frac{f / b}{3}\right) \xi(r), \quad \xi_{2}^{s}(s)=\frac{2 f / b}{3}[\xi(r)-\bar{\xi}(r)], \quad \xi_{4}^{s}(s)=0$.

\subsection{Estimator}

For our mock measurements of $\xi^{s}\left(s_{\perp}, s_{\|}\right)$, we utilize the Landy $\&$ Szalay (1993) estimator for cross correlations,

$\hat{\xi}^{s}\left(s_{\perp}, s_{\|}\right)=\frac{\left\langle\mathcal{D}_{\mathrm{v}} \mathcal{D}_{\mathrm{g}}\right\rangle-\left\langle\mathcal{D}_{\mathrm{v}} \mathcal{R}_{\mathrm{g}}\right\rangle-\left\langle\mathcal{R}_{\mathrm{v}} \mathcal{D}_{\mathrm{g}}\right\rangle+\left\langle\mathcal{R}_{\mathrm{v}} \mathcal{R}_{\mathrm{g}}\right\rangle}{\left\langle\mathcal{R}_{\mathrm{v}} \mathcal{R}_{\mathrm{g}}\right\rangle}$,

where the angled brackets signify normalized pair counts of voidcenter and galaxy positions in the data $\left(\mathcal{D}_{\mathrm{v}}, \mathcal{D}_{\mathrm{g}}\right)$ and corresponding random positions $\left(\mathcal{R}_{\mathrm{v}}, \mathcal{R}_{\mathrm{g}}\right)$ in bins of $s_{\perp}$ and $s_{\|}$. We chose a fixed binning in units of the effective void radius for each individual void and express all distances in units of $R$ as well. This allows one to coherently capture the characteristic topology of voids from a range of sizes including their boundaries in an ensemble-average sense. The resulting statistic is also referred to as a void stack or stacked void-galaxy cross-correlation function. We have generated the randoms via sampling from the redshift distributions of galaxies and voids as depicted in Fig. 1, but with ten times 

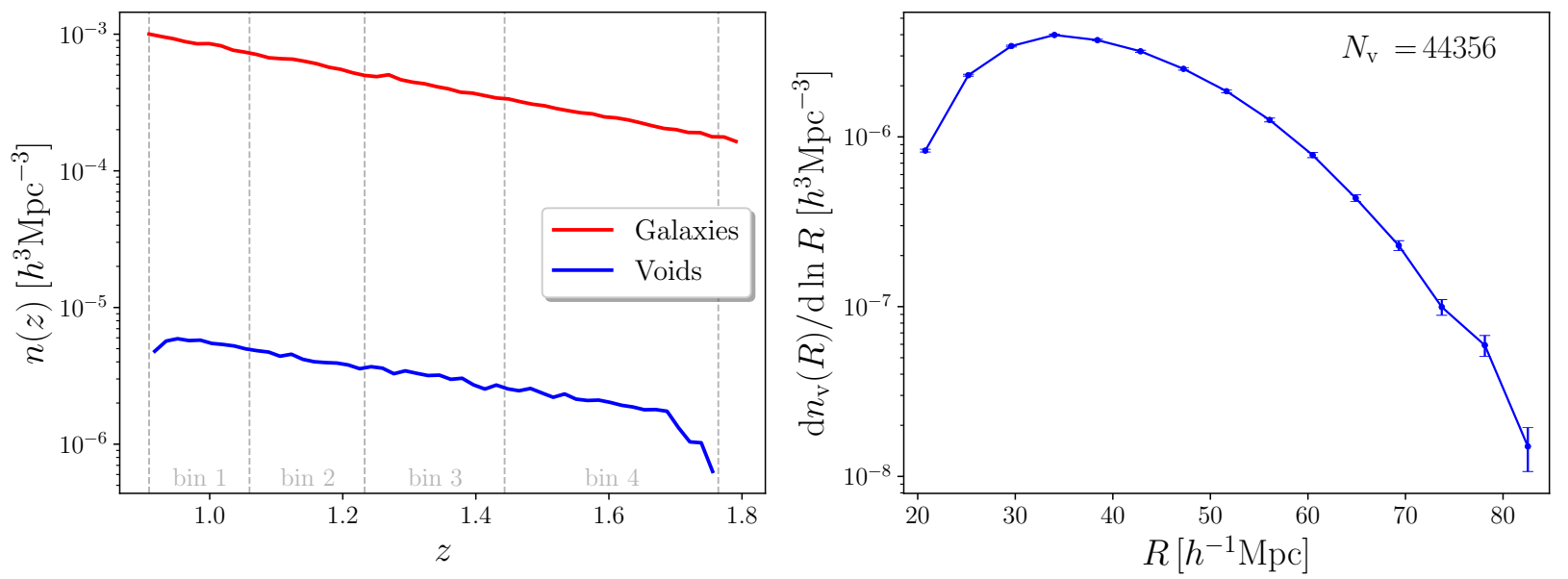

Fig. 1. Properties of tracers and voids in the Euclid Flagship mock catalog. Left: number density of spectroscopic galaxies and selected VIDE voids as function of redshift; vertical dashed lines indicate the redshift bins. Right: number density of the same VIDE voids as a function their effective radius $R$ (void size function) from the entire redshift range, encompassing a total of $N_{\mathrm{v}}=44356$ voids with $18.6 h^{-1} \mathrm{Mpc}<R<84.8 h^{-1} \mathrm{Mpc}$. Poisson statistics are assumed for the error bars. We refer to our companion paper for a detailed cosmological forecast based on the void size function (Contarini et al., in prep.).

the number of objects and without spatial clustering. We applied the same angular footprint as for the mock data and additionally assign an effective radius to every void random, drawn from the radius distribution of galaxy voids. The latter is used to express distances from void randoms in units of $R$, consistent with the stacking procedure of galaxy voids. The uncertainty of the estimated $\widehat{\xi}^{s}\left(s_{\perp}, s_{\|}\right)$is quantified by its covariance matrix:

$\hat{C}_{i j}=\left\langle\left(\hat{\xi}^{s}\left(\boldsymbol{s}_{i}\right)-\left\langle\hat{\xi}^{s}\left(\boldsymbol{s}_{i}\right)\right\rangle\right)\left(\hat{\xi}^{s}\left(\boldsymbol{s}_{j}\right)-\left\langle\hat{\xi}^{s}\left(\boldsymbol{s}_{j}\right)\right\rangle\right)\right\rangle$,

where angled brackets imply averaging over an ensemble of observations. The square root of the diagonal elements, $\hat{C}_{i i}$, are used as error bars on our measurements of $\hat{\xi}^{s}$. Although we can only observe one universe (respectively a single Flagship mock catalog), ergodicity allows us to estimate $\hat{C}_{i j}$ via spatial averaging over distinct patches. This naturally motivates the jackknife technique to be applied on the available sample of voids, which are non-overlapping. Therefore, we simply remove one void at a time in the estimator of $\xi^{s}$ from Eq. (17), which provides $N_{\mathrm{v}}$ jackknife samples. This approach has been tested on simulations and validated on mocks in previous analyses (Paz et al. 2013; Cai et al. 2016; Correa et al. 2019; Hamaus et al. 2020). It has further been shown that, in the limit of large sample sizes, the jackknife technique provides consistent covariance estimates compared to the ones obtained from many independent mock catalogs (Favole et al. 2021). Residual differences between the two methods indicate the jackknife approach to yield somewhat higher covariances, which renders our error forecast conservative.

\subsection{Model and likelihood}

As previously demonstrated in Hamaus et al. (2020), we include two additional nuisance parameters, $\mathcal{M}$ and $Q$, in the theory model of Eq. (7), enabling us to account for systematic effects. Here, $\mathcal{M}$ (monopole-like) is used as a free amplitude of the deprojected correlation function $\xi(r)$ in real space and $Q$ (quadrupole-like) is a free amplitude for the quadrupole term proportional to $\mu^{2}$. Here, we adopt a slightly modified, empirically motivated parametrization of this model, with enhanced coefficients for the Jacobian (second and third) terms in Eq. (7),

$\xi^{s}\left(s_{\perp}, s_{\|}\right)=\mathcal{M}\left\{\xi(r)+\frac{f}{b} \bar{\xi}(r)+2 Q \frac{f}{b} \mu^{2}[\xi(r)-\bar{\xi}(r)]\right\}$.

The parameter $\mathcal{M}$ adjusts for potential inaccuracies arising in the deprojection technique and a contamination of the void sample by spurious Poisson fluctuations, which can attenuate the amplitude of the monopole and quadrupole (Cousinou et al. 2019). The parameter $Q$ accounts for possible selection effects when voids are identified in anisotropic redshift space (Pisani et al. 2015b; Correa et al. 2021, 2022). For example, the occurrence of shell crossing and virialization affects the topology of void boundaries in redshift space (Hahn et al. 2015), resulting in the well-known finger-of-God (FoG) effect (Jackson 1972). In turn, this can enhance the Jacobian terms in Eq. (7), which motivates the empirically determined modification of their coefficients in Eq. (19). A similar result can be achieved by enhancing the values of $\mathcal{M}$ and $Q$, but keeping the original form of Eq. (7), which can be approximately understood as a redefinition of the nuisance parameters. However, the form of Eq. (19) is found to better describe the void-galaxy cross-correlation function in terms of goodness of fit, while at the same time yields nuisance parameters that are distributed more closely around values of one. This approach is akin to other empirical model extensions that have been proposed in the literature (e.g., Achitouv 2017; Paillas et al. 2021).

For the mapping from the observed separations $s_{\perp}$ and $s_{\|}$to $r$ and $\mu$, we use Eq. (3) together with Eq. (10) for the AP effect. This yields the following transformation between coordinates in real and redshift space,

$r_{\perp}=q_{\perp} s_{\perp}, \quad r_{\|}=q_{\|} s_{\|}\left[1-\frac{1}{3} \frac{f}{b} \mathcal{M} \bar{\xi}(r)\right]^{-1}$,

which can be solved via iteration to determine $r=\left(r_{\perp}^{2}+r_{\|}^{2}\right)^{1 / 2}$ and $\mu=r_{\|} / r$, starting from an initial value of $r=s$ (Hamaus et al. 2020). In practice, we express all separations in units of the observable effective radius $R$ of each void in redshift space, but noting that the AP effect yields $R^{*}=q_{\perp}^{2 / 3} q_{\|}^{1 / 3} R$ in the true cosmology (Hamaus et al. 2020; Correa et al. 2021). When expressing Eq. (20) in units of $R^{*}$, only ratios of $q_{\perp}$ and $q_{\|}$appear, which 
defines the AP parameter $\varepsilon=q_{\perp} / q_{\|}$. The latter is particularly well constrained via the AP test from standard spheres (Lavaux \& Wandelt 2012; Hamaus et al. 2015).

Finally, given the estimated data vector from Eq. (17), its covariance from Eq. (18), and the model from Eqs. (19) and (20), we can construct a Gaussian likelihood $L\left(\hat{\xi}^{s} \mid \boldsymbol{\Theta}\right)$ of the data $\hat{\xi}^{s}$ given the model parameter vector $\boldsymbol{\Theta}=(f / b, \varepsilon, \mathcal{M}, \boldsymbol{Q})$ as:

$\ln L\left(\hat{\xi}^{s} \mid \boldsymbol{\Theta}\right)=-\frac{1}{2} \sum_{i, j}\left(\hat{\xi}^{s}\left(\boldsymbol{s}_{i}\right)-\xi^{s}\left(\boldsymbol{s}_{i} \mid \boldsymbol{\Theta}\right)\right) \hat{C}_{i j}^{-1}\left(\hat{\xi}^{s}\left(\boldsymbol{s}_{j}\right)-\xi^{s}\left(\boldsymbol{s}_{j} \mid \boldsymbol{\Theta}\right)\right)$.

We apply the factor of Hartlap et al. (2007) to estimate the inverse covariance matrix, which amounts to a correction of at most $3 \%$ in our case. Because the model $\xi^{s}(s \mid \boldsymbol{\Theta})$ makes use of the data to calculate $\xi(r)$ via Eq. (5), it contributes its own covariance and becomes correlated with $\hat{\xi}^{s}(s)$. However, by treating the amplitude of $\xi(r)$ as the free parameter $\mathcal{M}$ in our model, we marginalize over its uncertainty. A correlation between model and data can only act to reduce the total covariance in our likelihood, so the resulting parameter errors can be regarded as upper limits in this respect. Due to their finite size, some voids extend beyond the boundaries of a given bin in redshift, which necessarily results in some degree of correlation between the parameter measurements at adjacent redshift bins. As a boundary effect, we expect this correlation to be small and hence neglect it here.

We use the affine-invariant Markov chain Monte Carlo (MCMC) ensemble sampler emcee (Foreman-Mackey et al. 2019) to sample the posterior probability distribution of all model parameters. The quality of the maximum-likelihood model (best fit) can be assessed via evaluation of the reduced $\chi^{2}$ statistic:

$\chi_{\text {red }}^{2}=-\frac{2}{N_{\text {d.o.f. }}} \ln L\left(\hat{\xi}^{s} \mid \boldsymbol{\Theta}\right)$,

with $N_{\text {d.o.f. }}=N_{\text {bin }}-N_{\text {par }}$ degrees of freedom, where $N_{\text {bin }}$ is the number of bins for the data and $N_{\text {par }}$ the number of model parameters. We use 18 bins per dimension for the 2D void-galaxy cross-correlation function, which yields $N_{\text {bin }}=324$. This number is high enough to accurately sample the scale dependence of $\hat{\xi}^{s}\left(s_{\perp}, s_{\|}\right)$, yet significantly smaller than the available number of voids per redshift bin, ensuring sufficient statistics for the estimation of its covariance matrix. With $N_{\mathrm{par}}=4$, this implies $N_{\text {d.o.f. }}=320$.

\subsection{Deprojection and fit}

In order to evaluate our model from Eq. (19), we need to obtain the real-space correlation function $\xi(r)$, which can be calculated via an inverse Abel transform of the projected redshift-space correlation function $\xi_{\mathrm{p}}^{s}\left(s_{\perp}\right)$, following Eq. (5). We estimate the function $\xi_{\mathrm{p}}^{s}\left(s_{\perp}\right)$ directly via line-of-sight integration of $\hat{\xi}^{s}\left(s_{\perp}, s_{\|}\right)$, as in the first equality of Eq. (6), and use a cubic spline to interpolate both $\xi_{\mathrm{p}}^{s}\left(s_{\perp}\right)$ and $\xi(r)$. The results are presented in Fig. 2 for each of our four redshift bins. Thanks to the large number of voids in each bin, the statistical noise is low and enables a smooth deprojection of the data. Some residual noise can be noted at the innermost bins, namely, for small separations from the void center, which causes the deprojection and the subsequent spline interpolation to be less accurate (Pisani et al. 2014; Hamaus et al. 2020). For this reason, we omit the first radial bin of the data in our model fits below, but we checked that even discarding the central three bins of $s, s_{\perp}$, and $s_{\|}$in our analysis yields consistent results. From the full Euclid footprint of about three times the size of an octant, the residual statistical noise of this procedure will be reduced further, so our mock analysis can be considered conservative in this regard.

We also plot the monopole of the redshift-space correlation function, which nicely follows the shape of the deprojected $\xi(r)$, as expected from Eq. (16). Moreover, our model from Eqs. (19) and (20) provides a very accurate fit to this monopole everywhere apart from its innermost bins, implying that any residual errors in the model remain negligibly small in that regime. We notice an increasing amplitude of all correlation functions towards lower redshift, partly reflecting the growth of overdensities along the void walls, while their interior is gradually evacuated. The increase in mean effective radius with redshift is not of dynamical origin, it is a consequence of the declining galaxy density $n_{\mathrm{g}}(z)$, see Fig. 1 . A higher space density of tracers enables the identification of smaller voids.

We minimize the log-likelihood of Eq. (21) by varying the model parameters to find the best-fit model to the mock data. As a data vector, we used the $2 \mathrm{D}$ void-galaxy cross-correlation $\hat{\xi}^{s}\left(s_{\perp}, s_{\|}\right)$, which contains the complete information on dynamic and geometric distortions from all multipole orders. However, we checked that our pipeline yields consistent constraints when only considering the three lowest even multipoles, $\hat{\xi}_{\ell}^{s}(s)$, of order $\ell=0,2,4$ as our data vector. The results are shown in Fig. 3 for our four consecutive redshift bins. In each case, we find extraordinary agreement between the model and the data, which is further quantified by the reduced $\chi^{2}$ being so close to unity in all bins. Again, it is possible to perceive the slight deepening of voids over time and the agglomeration of matter in their surroundings. The multipoles shown in the right column of Fig. 3 complement this view, with both monopole and quadrupole enhancing their amplitudes during void evolution, and they exhibit an excellent agreement with the model. The quadrupole vanishes towards the central void region and the model mismatch in the first few bins of the monopole has negligible impact here, as most of the anisotropic information originates from larger scales. In addition, the hexadecapole remains consistent with zero at all times, in accordance with Eq. (16).

\subsection{Parameter constraints}

Performing a full MCMC run for each redshift bin, we obtain the posteriors for our model parameters as shown in Fig. 4. We observe a similar correlation structure as in the previous BOSS analysis of Hamaus et al. (2020). Namely, a weak correlation between $f / b$ and $\varepsilon$, and a strong anti-correlation between $f / b$ and $\mathcal{M}$. Overall, the $68 \%$ confidence regions for $f / b$ and $\varepsilon$ agree well with the expected input values, shown as dashed lines. In a $\Lambda \mathrm{CDM}$ cosmology, the linear growth rate is given by

$f(z) \simeq\left[\frac{\Omega_{\mathrm{m}}(1+z)^{3}}{H^{2}(z) / H_{0}^{2}}\right]^{\gamma}$,

with a growth index of $\gamma \simeq 0.55$ (Lahav et al. 1991; Linder 2005). We take the Flagship measurements of the large-scale linear bias $b$ from our companion paper (Contarini et al., in prep.), which uses CAMB (Lewis et al. 2000) to calculate the dark-matter correlation function to compare with the estimated galaxy autocorrelation function (see Marulli et al. 2013, 2018, for details). As we are using the correct input cosmology of Flagship to convert angles and redshifts to comoving distances following Eq. (9), we have $\varepsilon=1$ as fiducial value.

For the values of the nuisance parameters, $\mathcal{M}$ and $Q$, we do not have any specific expectation, but we set their defaults 

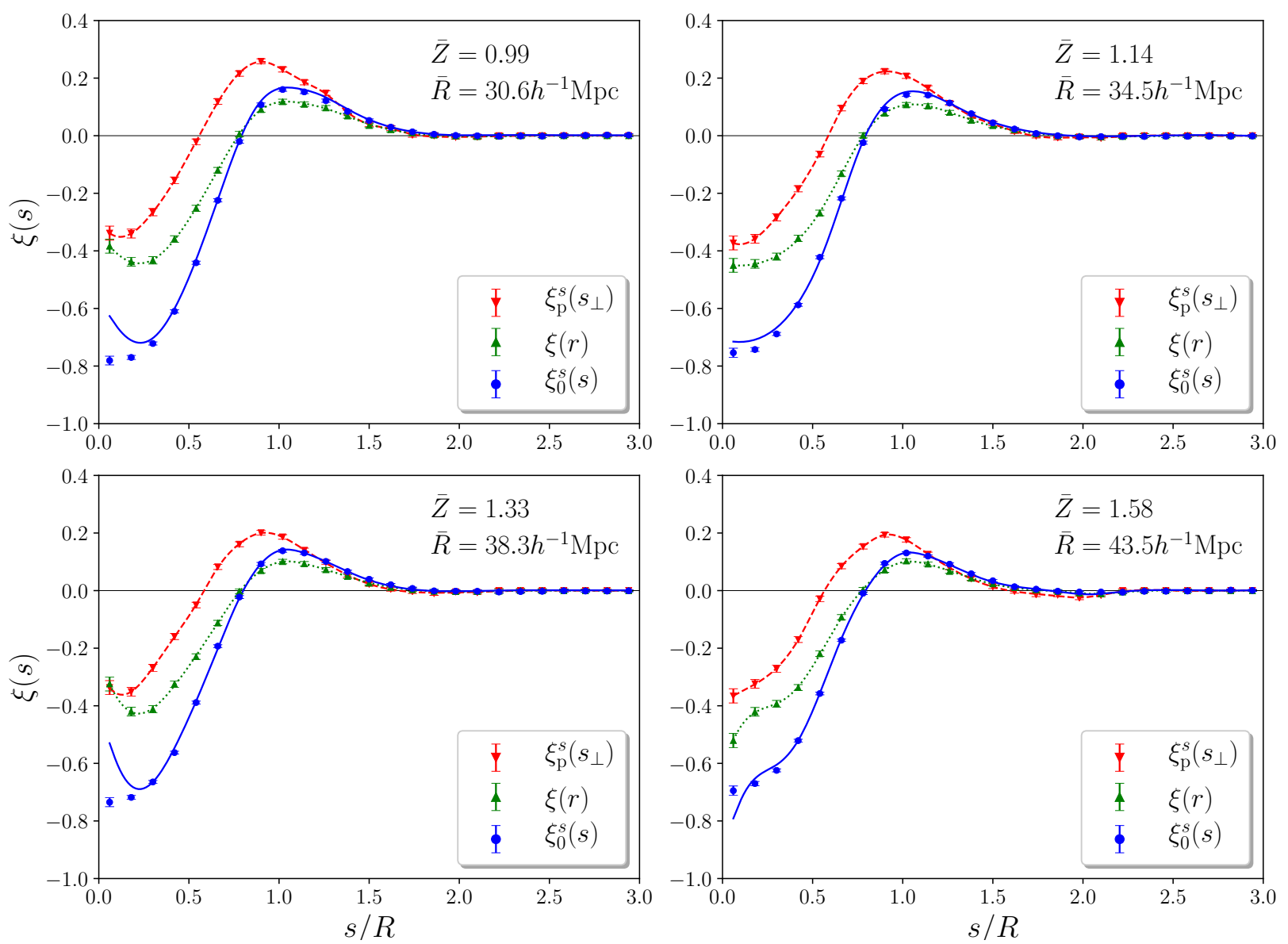

Fig. 2. Projected void-galaxy cross-correlation function $\xi_{\mathrm{p}}^{s}\left(s_{\perp}\right)$ in redshift space (red wedges, interpolated with dashed line) and its real-space counterpart $\xi(r)$ in 3D after deprojection (green triangles interpolated with dotted line). The redshift-space monopole $\xi_{0}^{s}(s)$ (blue dots) and its best-fit model based on Eqs. (19) and (20) is shown for comparison (solid line). Adjacent bins in redshift increase from the upper left to the lower right, with mean void redshift, $\bar{Z}$, and effective radius, $\bar{R}$, as indicated in each panel.

to unity as well. We also find their posteriors to be distributed around values of one, although their mean can deviate more than one standard deviation from that default value. However, the distributions of the nuisance parameters are not relevant for the cosmological interpretation of the posterior, as they can be marginalized over. The relative precision on $f / b$ ranges between $7.3 \%$ and $8.0 \%$, while the one on $\varepsilon$ is between $0.87 \%$ and $0.91 \%$. This precision corresponds to a survey area of one octant of the sky, but the footprint covered by Euclid will be roughly three times as large. Therefore, one can expect these numbers to decrease by a factor of $\sqrt{3}$ to yield about $4 \%$ accuracy on $\mathrm{f} / \mathrm{b}$ and $0.5 \%$ on $\varepsilon$ per redshift bin.

The attainable precision can even further be increased via a calibration strategy. Hamaus et al. (2020) have shown that this is possible when the model ingredients $\xi(r), \mathcal{M}$, and $Q$ are taken from external sources, instead of being constrained by the data itself, for example, from a large number of high-fidelity survey mocks. However, we emphasize that this practice introduces a prior dependence on the assumed model parameters in the mocks, so it underestimates the final uncertainty on cosmology and may yield biased results. We also note that survey mocks are typically designed to reproduce the two-point statistics of galaxies on large scales, but are not guaranteed to provide void statistics at a similar level of accuracy.

Nevertheless, for the sake of completeness we investigate the achievable precision when fixing the nuisance parameters to their best-fit values in the full analysis, while still inferring $\xi(r)$ via deprojection of the data as before. We note that this is an arbitrary choice of calibration, in practice, the values of $\mathcal{M}$ and $Q$ will depend on the type of mocks considered. The resulting posteriors on $f / b$ and $\varepsilon$ are shown in Fig. 5. The calibrated analysis yields a relative precision of $1.3 \%$ to $1.8 \%$ on $f / b$ and $0.72 \%$ to $0.75 \%$ on $\varepsilon$. Compared to the calibration-independent analysis, this amounts to an improvement by up to a factor of about 5 for constraints on $f / b$ and 1.2 for $\varepsilon$. Extrapolated to the full survey area accessible to Euclid, this corresponds to a precision of roughly $1 \%$ on $f / b$ and $0.4 \%$ on $\varepsilon$ per redshift bin. As expected, these calibrated constraints are more prone to be biased with respect to the underlying cosmology, as evident from Fig. 5 given our choice of calibration. It is also interesting to note that $f / b$ and $\varepsilon$ are less correlated in the calibrated analysis, since their partial degeneracy with the nuisance parameter $\mathcal{M}$ is removed.

We summarize all of our results in Table 1. The constraints on $f \sigma_{8}$ and $D_{\mathrm{M}} H$ are derived from the posteriors on $f / b$ and $\varepsilon$. In the former case, we assume $\xi(r) \propto b \sigma_{8}$ and, hence, we multiply $f / b$ by the underlying value of $b \sigma_{8}$ in the Flagship mock, which also assumes the relative precision on $f / b$ and $f \sigma_{8}$ to be the same. Moreover, we neglect the dependence on $h$ that enters in the definition of $\sigma_{8}$ and should be marginalized over (Sánchez 2020). For the latter case, we multiply $\varepsilon$ by the fiducial $D_{\mathrm{M}} H$, following Eq. (11). The results on $f \sigma_{8}$ and $D_{\mathrm{M}} H$ from 

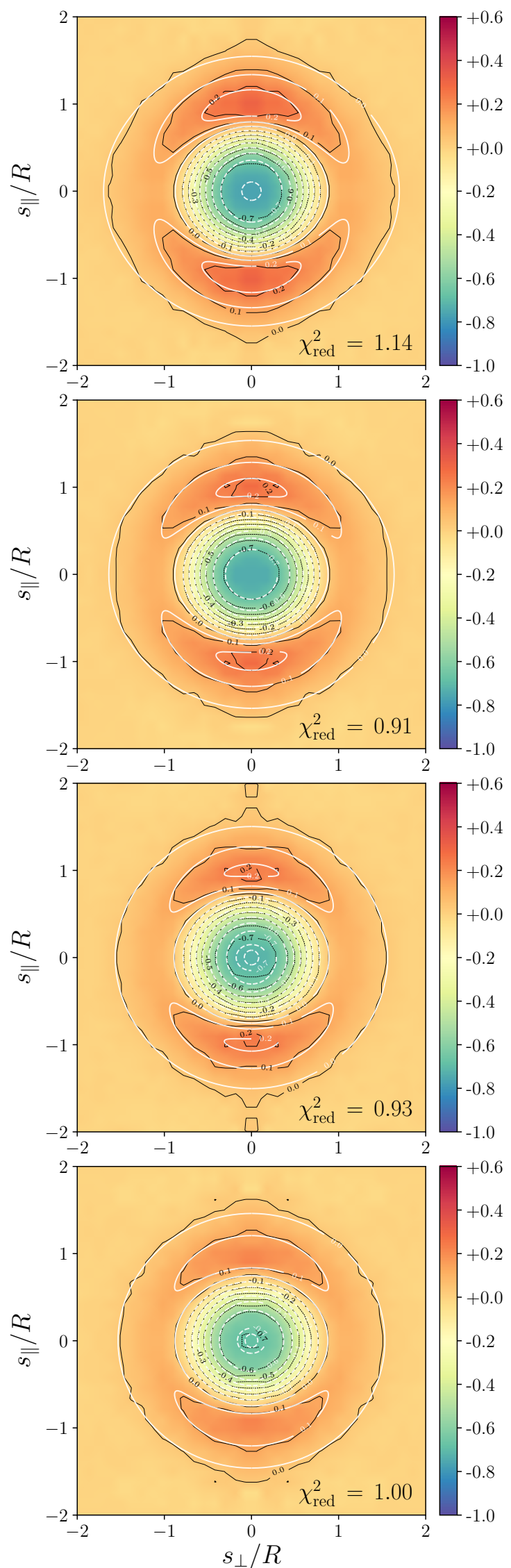
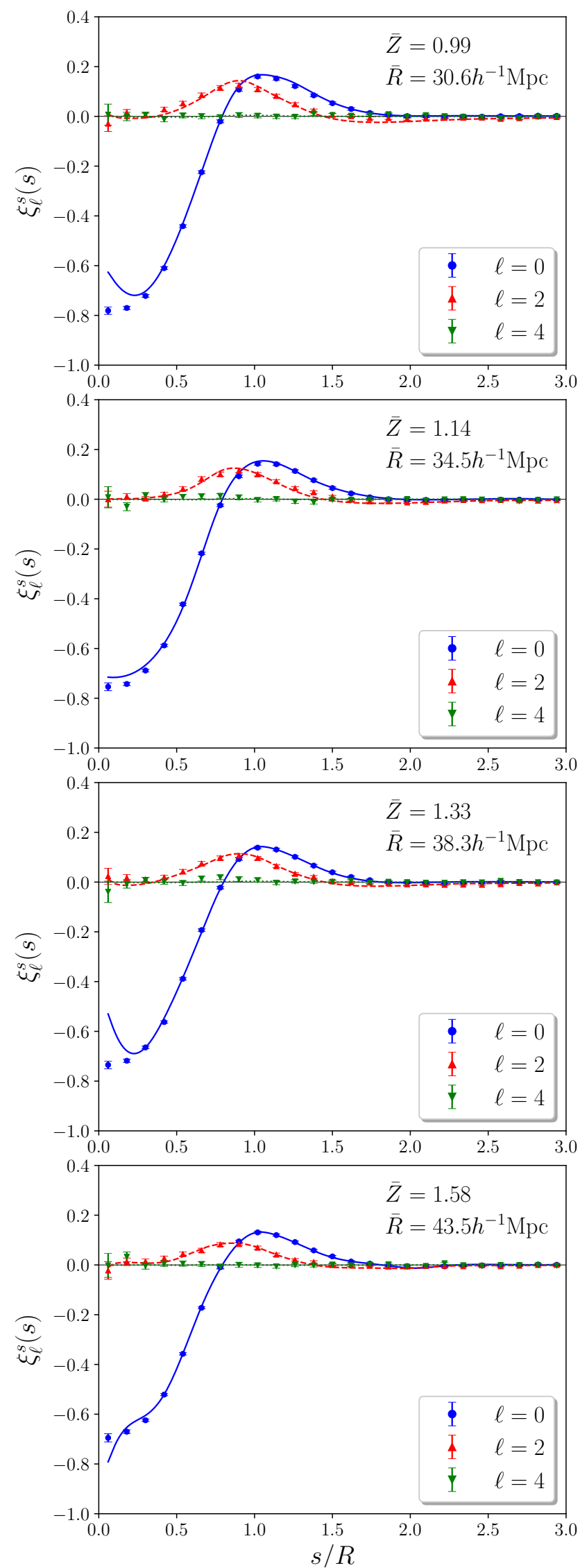

Fig. 3. Stacked void-galaxy cross-correlation function in redshift space. Left: $\xi^{s}\left(s_{\perp}, s_{\|}\right)$in 2D (color scale with black contours) and its best-fit model from Eqs. (19) and (20) (white contours). Right: monopole (blue dots), quadrupole (red triangles) and hexadecapole (green wedges) of $\xi^{s}\left(s_{\perp}, s_{\|}\right)$and best-fit model (solid, dashed, dotted lines). The mean void redshift, $\bar{Z}$, and effective radius, $\bar{R}$, of each redshift bin are indicated. 

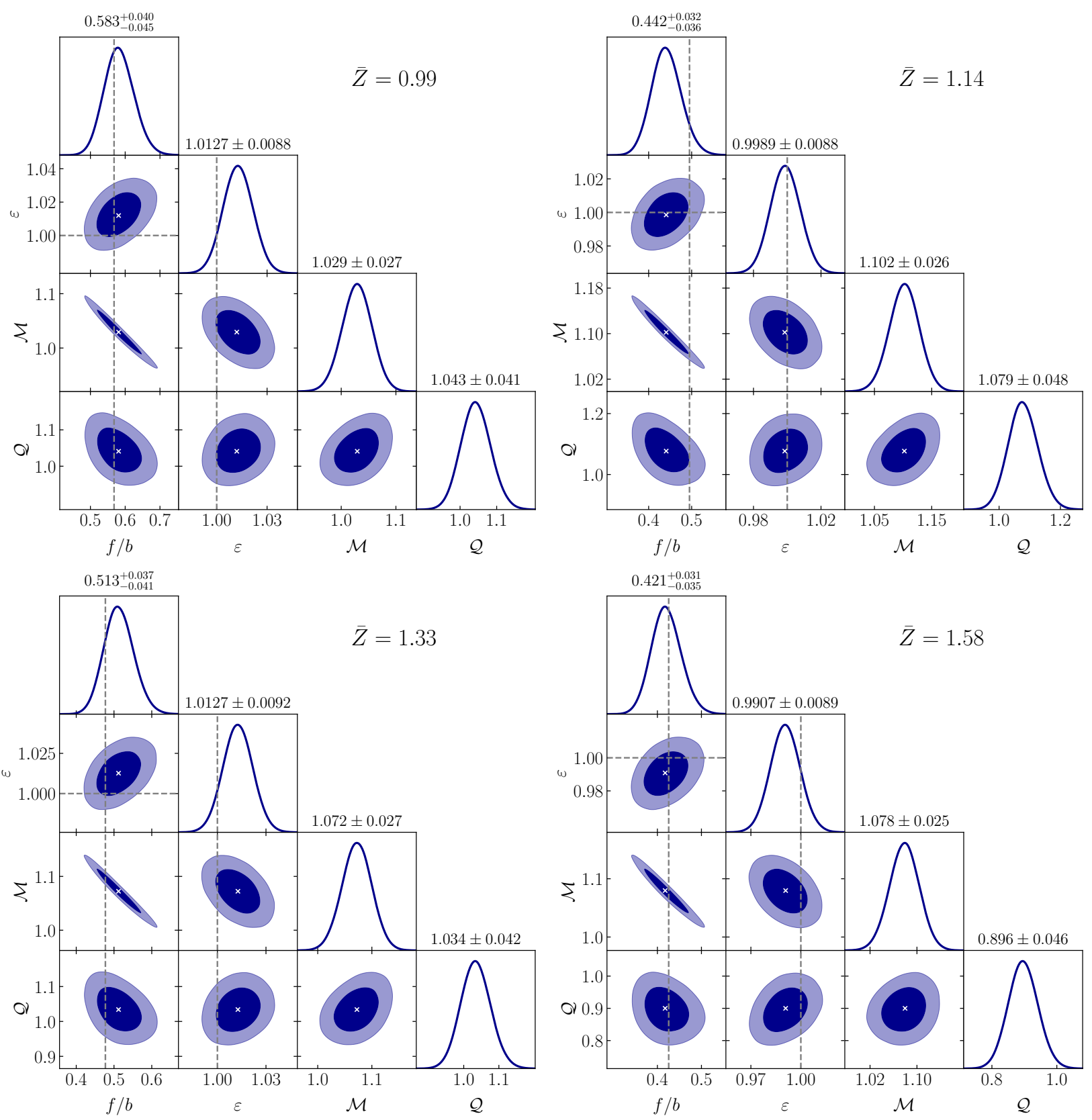

Fig. 4. Posterior probability distribution of the model parameters that enter in Eqs. (19) and (20), obtained via MCMC from the data shown in the left of Fig. 3. Dark and light shaded areas represent 68\% and 95\% confidence regions with a cross marking the best fit, dashed lines indicate fiducial values of the RSD and AP parameters. The top of each column states the mean and standard deviation of the 1D marginal distributions. Adjacent bins in void redshift with mean value $\bar{Z}$ increase from the upper left to the lower right, as indicated.
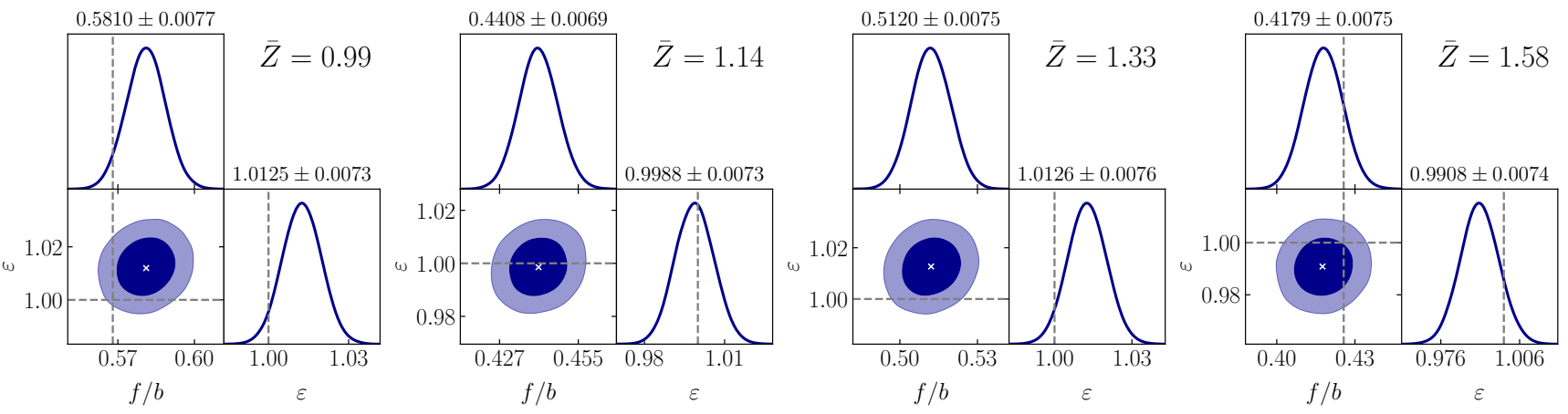

Fig. 5. Posterior probability distribution of the model parameters that enter in Eqs. (19) and (20). Details are the same as in Fig. 4, but with a fixing (calibration) of the nuisance parameters $\mathcal{M}$ and $Q$ to their best-fit values. Redshift increases from left to right, as indicated. 
Table 1. RSD and AP parameter constraints.

\begin{tabular}{lcccccccc}
\hline \hline Data & $Z_{\min }$ & $Z_{\max }$ & $\bar{Z}$ & $b$ & $f / b$ & $f \sigma_{8}$ & $\varepsilon$ & $D_{\mathrm{M}} H / c$ \\
\hline Euclid voids & 0.91 & 1.06 & 0.99 & 1.54 & $0.5827 \pm 0.0427$ & $0.4544 \pm 0.0333$ & $1.0127 \pm 0.0088$ & $1.3627 \pm 0.0119$ \\
(independent) & 1.06 & 1.23 & 1.14 & 1.81 & $0.4416 \pm 0.0346$ & $0.3784 \pm 0.0296$ & $0.9989 \pm 0.0088$ & $1.6391 \pm 0.0144$ \\
& 1.23 & 1.44 & 1.33 & 1.92 & $0.5132 \pm 0.0394$ & $0.4320 \pm 0.0331$ & $1.0127 \pm 0.0092$ & $2.0517 \pm 0.0186$ \\
& 1.44 & 1.76 & 1.58 & 2.20 & $0.4205 \pm 0.0337$ & $0.3689 \pm 0.0296$ & $0.9907 \pm 0.0089$ & $2.5543 \pm 0.0230$ \\
\hline Euclid voids & 0.91 & 1.06 & 0.99 & 1.54 & $0.5810 \pm 0.0076$ & $0.4531 \pm 0.0060$ & $1.0125 \pm 0.0073$ & $1.3624 \pm 0.0098$ \\
(calibrated) & 1.06 & 1.23 & 1.14 & 1.81 & $0.4408 \pm 0.0069$ & $0.3777 \pm 0.0059$ & $0.9988 \pm 0.0073$ & $1.6389 \pm 0.0120$ \\
& 1.23 & 1.44 & 1.33 & 1.92 & $0.5120 \pm 0.0075$ & $0.4310 \pm 0.0064$ & $1.0127 \pm 0.0076$ & $2.0518 \pm 0.0153$ \\
& 1.44 & 1.76 & 1.58 & 2.20 & $0.4179 \pm 0.0075$ & $0.3666 \pm 0.0066$ & $0.9908 \pm 0.0074$ & $2.5546 \pm 0.0191$ \\
\hline
\end{tabular}

Notes. Forecasted constraints on RSD and AP parameters (mean values with $68 \%$ confidence intervals) from VIDE voids in the Euclid Flagship mock catalog (top rows). Results are given in four redshift bins with minimum, maximum, and mean void redshift $Z_{\min }, Z_{\mathrm{max}}, \bar{Z}$, and large-scale galaxy bias $b$. All uncertainties correspond to one octant of the sky, but the expected precision from the full Euclid footprint is a factor of about $\sqrt{3}$ higher. The bottom rows show more optimistic constraints after performing a calibration of the nuisance parameters in the model.

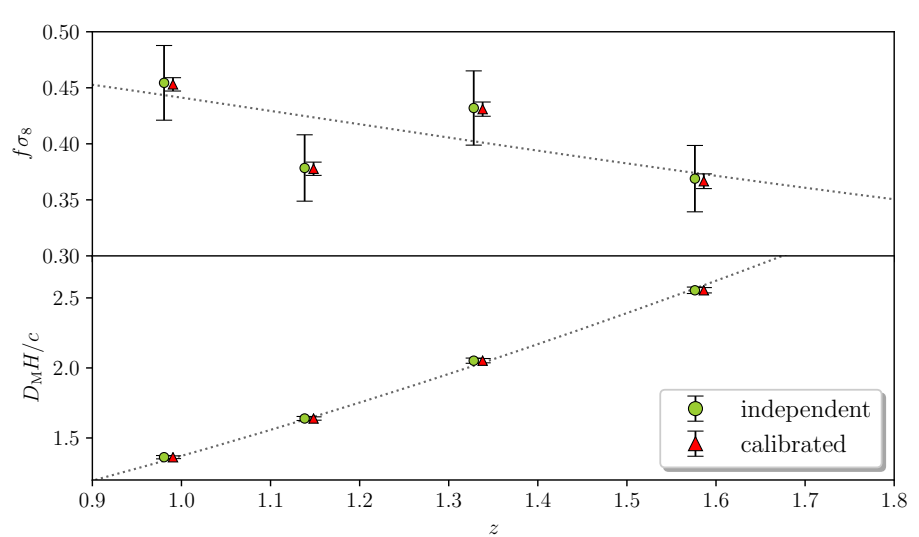

Fig. 6. Measurement of $f \sigma_{8}$ and $D_{\mathrm{M}} H$ from VIDE voids in the Euclid Flagship catalog as a function of redshift $z$. The marker style distinguishes between a fully model-independent approach (green circles) and an analysis with calibrated nuisance parameters $\mathcal{M}$ and $Q$ (red triangles) using external sources, such as simulations or mocks. Dotted lines indicate the Flagship input cosmology, the markers are slightly shifted horizontally for visibility. These results are based on one octant of the sky, the expected precision from the full Euclid footprint is a factor of about $\sqrt{3}$ higher.

both model-independent and calibrated analyses are also shown in Fig. 6.

\section{Discussion}

The measurements of $f \sigma_{8}$ and $D_{\mathrm{M}} H$ as a function of redshift can be used to constrain cosmological models. For example, an inversion of Eq. (12) provides $\Omega_{\Lambda}=1-\Omega_{\mathrm{m}}$, the only free parameter of the product $D_{\mathrm{M}} H$ in a flat $\Lambda$ CDM cosmology. Using our Flagship mock measurements of $D_{\mathrm{M}} H$ we sample the joint posterior on $\Omega_{\Lambda}$ from all of our redshift bins combined. Considering the full Euclid footprint to be approximately three times the size of our Flagship mock catalog, we scale the errors on $D_{\mathrm{M}} H$ by a factor of $1 / \sqrt{3}$ and center its mean values to the input cosmology of Flagship. The resulting posterior yields $\Omega_{\Lambda}=0.6809 \pm 0.0048$, in the model-independent, and $\Omega_{\Lambda}=0.6810 \pm 0.0039$, in the calibrated case from the analysis of Euclid voids alone. The corresponding result obtained by Planck in 2018 (Planck Collaboration VI 2020) is $\Omega_{\Lambda}=0.6847 \pm 0.0073$, including cosmic microwave background (CMB) lensing and
$\Omega_{\Lambda}=0.6889 \pm 0.0056$, when combined with BOSS BAO data (Alam et al. 2017). The main cosmological probes of Euclid, when altogether combined, are forecasted to achieve a $1 \sigma$ uncertainty of 0.0071 on $\Omega_{\Lambda}$ in a pessimistic scenario and 0.0025 in an optimistic case (Euclid Collaboration 2020). The expected precision on $\Omega_{\Lambda}$ from the analysis of Euclid voids alone will hence likely match the level of precision from Planck and the combined main Euclid probes. The left panel of Fig. 7 provides a visual comparison of the constraining power on $\Omega_{\Lambda}$ from the mentioned experiments, including the one previously obtained from BOSS voids in Hamaus et al. (2020).

Furthermore, in Euclid we aim to explore cosmological models beyond $\Lambda \mathrm{CDM}$. One minimal extension is to replace the cosmological constant, $\Lambda$, by a more general form of dark energy with density $\Omega_{\mathrm{de}}$, and a constant equation-of-state parameter, $w$. This modifies the Hubble function in Eq. (12) to

$H(z)=H_{0} \sqrt{\left(1-\Omega_{\mathrm{de}}\right)(1+z)^{3}+\Omega_{\mathrm{de}}(1+z)^{3(1+w)}}$,

which reduces to the case of flat $\Lambda \mathrm{CDM}$ for $w=-1$. Using our rescaled and recentered mock measurements of $D_{\mathrm{M}} H$, we can thus infer the posterior distribution of the parameter pair $\left(\Omega_{\mathrm{de}}, w\right)$. The result is shown in the right panel of Fig. 7 for both the model-independent and the calibrated analysis, assuming flat priors with $\Omega_{\mathrm{de}} \in[0,1]$ and $w \in[-10,10]$. We observe a mild degeneracy between $\Omega_{\mathrm{de}}$ and $w$, which can be mitigated in the calibrated case. However, this may come at the price of an increased bias from the true cosmology. Nevertheless, these parameter constraints are extremely competitive, yielding $w=-1.01_{-0.08}^{+0.10}$ and, hence, a relative precision of about $9 \%$ in the calibrated scenario. In the model-independent analysis, we still obtain $w=-1.01_{-0.10}^{+0.12}$ with a relative precision of $11 \%$. The constraints on $\Omega_{\mathrm{de}}$ are similar to the ones on $\Omega_{\Lambda}$ from above. We note that this result is in remarkable agreement with the early Fisher forecast of Lavaux \& Wandelt (2012), corroborating the robustness of the AP test with voids. The Planck Collaboration VI (2020) obtain $w=-1.57_{-0.40}^{+0.50}$ including CMB lensing in the same $w \mathrm{CDM}$ model and a combination with BAO yields a similar precision of about $10 \%$, with $w=-1.04_{-0.10}^{+0.10}$.

The right panel of Fig. 7 provides a demonstration of how cosmic voids by themselves constrain the properties of dark energy, without the inclusion of external priors, observables, or mock data. A combination with other probes, such as void abundance (Contarini et al., in prep.), cluster abundance (Sahlén 2019), BAO (Nadathur et al. 2019), CMB, or weak lensing 

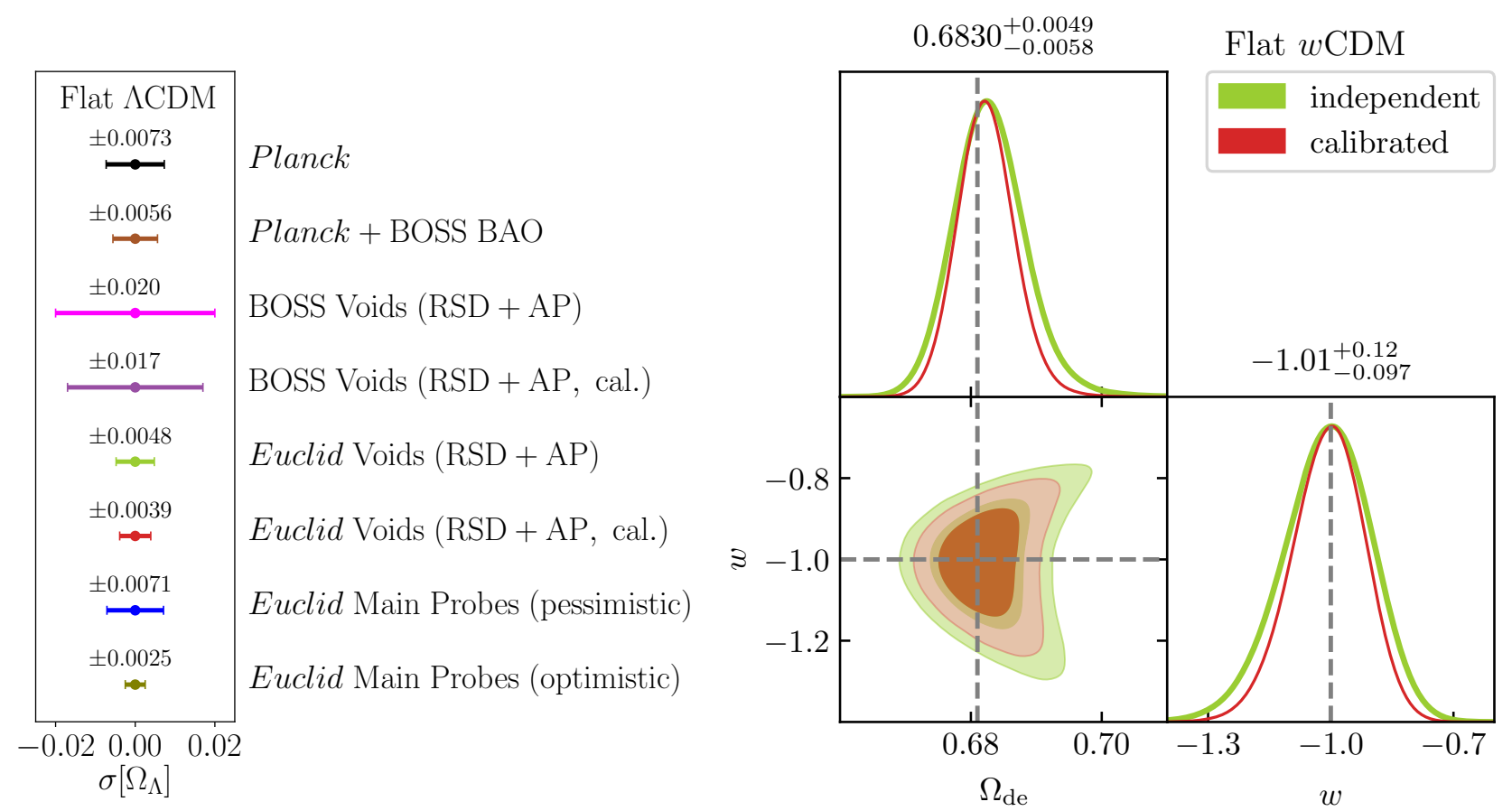

Fig. 7. Forecast for cosmological constraints on dark energy parameters. Left: comparison of the constraining power on $\Omega_{\Lambda}$ in a flat $\Lambda$ CDM cosmology from Planck 2018 alone (Planck Collaboration VI 2020) and when combined with BOSS BAO data (Alam et al. 2017). Below, constraints as obtained with BOSS voids via RSD and AP (Hamaus et al. 2020), as expected from Euclid voids (this work), and as expected from Euclid's main cosmological probes combined (Euclid Collaboration 2020). Calibrated constraints from voids are indicated by the abbreviation "cal." and the Euclid main probes distinguish between "optimistic" and "pessimistic" scenarios. Right: predicted constraints from Euclid voids on dark energy content $\Omega_{\mathrm{de}}$ and its equation-of-state parameter $w$ in a flat $w \mathrm{CDM}$ cosmology. Both the model-independent (green) and the calibrated results (red) are shown, dashed lines indicate the input cosmology. Mean parameter values and their $68 \%$ confidence intervals for the modelindependent case are shown at the top of each panel.

(Bonici et al., in prep.) will, of course, provide substantial gains. This is particularly relevant when extended cosmological models are considered to enable the breaking of parameter degeneracies. For example, it concerns models with a redshift-dependent equation of state $w(z)$, nonzero curvature, or massive neutrinos. In those cases, however, the calibrated approach is prone to be biased towards the fiducial cosmology adopted in the synthetic mock data used for calibration, which commonly assumes a flat $\Lambda \mathrm{CDM}$ model. In order to avoid the emergence of confirmation bias, we advocate the more conservative model-independent approach, even if it comes at the price of a somewhat reduced statistical precision.

In our analysis, we have neglected observational systematics that are expected to arise in Euclid data, such as spectral line misidentification due to interlopers that can lead to catastrophic redshift errors (Pullen et al. 2016; Leung et al. 2017; Massara et al. 2021). However, this effect mainly impacts the amplitude of multipoles (Addison et al. 2019; Grasshorn Gebhardt et al. 2019), so we anticipate it to be at least partially captured by the nuisance parameters in our model. We leave a more detailed investigation on the impact of survey systematics to a future work.

\section{Conclusion}

In this work, we investigate the prospects for performing a cosmological analysis using voids extracted from the spectroscopic galaxy sample of the Euclid Survey. The method we applied is based on the observable distortions of average void shapes via RSD and the AP effect. Our forecast relies on one light cone octant $\left(5157 \mathrm{deg}^{2}\right)$ of the Flagship simulation covering a red- shift range of $0.9<z<1.8$, which provides the most realistic mock galaxy catalog available for this purpose to date. Exploiting a deprojection technique and assuming linear mass conservation allows us to accurately model the anisotropic void-galaxy cross-correlation function in redshift space. We explore the likelihood of the mock data given this model via MCMCs and obtain the posterior distributions for our model parameters: the ratio of growth rate and bias $f / b$, and the geometric AP distortion $\varepsilon$. Two additional nuisance parameters, $\mathcal{M}$ and $Q$, are used to account for systematic effects in the data; they can either be marginalized over (model-independent approach) or calibrated via external sources, such as survey mocks (calibrated approach). After the conversion of our model parameters to the combinations $f \sigma_{8}$ and $D_{\mathrm{M}} H$, we forecast the attainable precision of their measurement with voids in Euclid.

We expect a relative precision of about $4 \%(1 \%)$ on $f \sigma_{8}$ and $0.5 \%(0.4 \%)$ on $D_{\mathrm{M}} H$ without (with) model calibration for each of our four redshift bins. This level of precision will enable very competitive constraints on cosmological parameters. For example, it yields a $0.7 \%(0.6 \%)$ constraint on $\Omega_{\Lambda}$ in a flat $\Lambda \mathrm{CDM}$ cosmology and a $11 \%(9 \%)$ constraint on the equation-of-state parameter $w$ for dark energy in $w \mathrm{CDM}$ from the AP test with voids alone. A combination with other void statistics, or the main cosmological probes of Euclid, such as galaxy clustering and weak lensing, will enable considerable improvements in accuracy and allow for the exploration of a broader range of extended cosmological models with a larger scope of parameters.

Acknowledgements. NH, GP and JW are supported by the Excellence Cluster ORIGINS, which is funded by the Deutsche Forschungsgemeinschaft (DFG, German Research Foundation) under Germany's Excellence Strategy - EXC2094 - 390783311. MA, MCC and SE are supported by the eBOSS ANR grant 
(under contract ANR-16-CE31-0021) of the French National Research Agency, the OCEVU LABEX (Grant No. ANR-11-LABX-0060) and the A*MIDEX project (Grant No. ANR-11-IDEX-0001-02) funded by the Investissements d'Avenir French government program, and by CNES, the French National Space Agency. AP is supported by NASA ROSES grant 12-EUCLID12-0004, and NASA grant 15-WFIRST15-0008 to the Nancy Grace Roman Space Telescope Science Investigation Team "Cosmology with the High Latitude Survey". GL is supported by the ANR BIG4 project, grant ANR-16-CE23-0002 of the French Agence Nationale de la Recherche. PN is funded by the Centre National d'Etudes Spatiales (CNES). We acknowledge use of the Python libraries NumPy (Harris et al. 2020), SciPy (Virtanen et al. 2020), Matplotlib (Hunter 2007), Astropy (Astropy Collaboration 2013, 2018), emcee (Foreman-Mackey et al 2019), GetDist (Lewis 2019), healpy (Górski et al. 2005; Zonca et al. 2019), and PyAbel (Hickstein et al. 2019). This work has made use of CosmoHub (Carretero et al. 2017; Tallada et al. 2020). CosmoHub has been developed by the Port d'Informació Científica (PIC), maintained through a collaboration of the Institut de Física d'Altes Energies (IFAE) and the Centro de Investigaciones Energéticas, Medioambientales y Tecnológicas (CIEMAT) and the Institute of Space Sciences (CSIC \& IEEC), and was partially funded by the "Plan Estatal de Investigación Científica y Técnica y de Innovación” program of the Spanish government. The Euclid Consortium acknowledges the European Space Agency and a number of agencies and institutes that have supported the development of Euclid, in particular the Academy of Finland, the Agenzia Spaziale Italiana, the Belgian Science Policy, the Canadian Euclid Consortium, the Centre National d'Etudes Spatiales, the Deutsches Zentrum für Luft- und Raumfahrt, the Danish Space Research Institute, the Fundação para a Ciência e a Tecnologia, the Ministerio de Economia y Competitividad, the National Aeronautics and Space Administration, the Netherlandse Onderzoekschool Voor Astronomie, the Norwegian Space Agency, the Romanian Space Agency, the State Secretariat for Education, Research and Innovation (SERI) at the Swiss Space Office (SSO), and the United Kingdom Space Agency. A complete and detailed list is available on the Euclid website (http: //www . euclid-ec.org).

\section{References}

Abel, N. H. 1842, in Oeuvres Completes, eds. L. Sylow, \& S. Lie (New York Johnson Reprint Corp.), 27

Abel, T., Hahn, O., \& Kaehler, R. 2012, MNRAS, 427, 61

Achitouv, I. 2016, Phys. Rev. D, 94, 103524

Achitouv, I. 2017, Phys. Rev. D, 96, 083506

Achitouv, I. 2019, Phys. Rev. D, 100, 123513

Addison, G. E., Bennett, C. L., Jeong, D., Komatsu, E., \& Weiland, J. L. 2019, $\mathrm{ApJ}, 879,15$

Alam, S., Ata, M., Bailey, S., et al. 2017, MNRAS, 470, 2617

Alam, S., Aubert, M., Avila, S., et al. 2021a, Phys. Rev. D, 103, 083533

Alam, S., Aviles, A., Bean, R., et al. 2021b, JCAP, 11, 050

Albrecht, A., Bernstein, G., Cahn, R., et al. 2006, ArXiv e-prints [arXiv:astro-ph/0609591]

Alcock, C., \& Paczynski, B. 1979, Nature, 281, 358

Alonso, D., Hill, J. C., Hložek, R., \& Spergel, D. N. 2018, Phys. Rev. D, 97 063514

Amendola, L., Appleby, S., Avgoustidis, A., et al. 2018, Liv. Rev. Relativ., 21, 2 Astropy Collaboration (Robitaille, T. P., et al.) 2013, A\&A, 558, A33

Astropy Collaboration (Price-Whelan, A. M., et al.) 2018, AJ, 156, 123

Aubert, M., Cousinou, M.-C., Escoffier, S., et al. 2020, ArXiv e-prints [arXiv:2007.09013]

Baker, T., Clampitt, J., Jain, B., \& Trodden, M. 2018, Phys. Rev. D, 98, 023511

Baldi, M., \& Villaescusa-Navarro, F. 2018, MNRAS, 473, 3226

Banerjee, A., \& Dalal, N. 2016, JCAP, 11, 015

Barreira, A., Cautun, M., Li, B., Baugh, C. M., \& Pascoli, S. 2015, JCAP, 2015, 028

Bayer, A. E., Villaescusa-Navarro, F., Massara, E., et al. 2021, ApJ, 919, 24

Behroozi, P. S., Wechsler, R. H., \& Wu, H.-Y. 2013, ApJ, 762, 109

Bertschinger, E. 1985, ApJS, 58, 1

Blanton, M. R., Hogg, D. W., Bahcall, N. A., et al. 2003, ApJ, 592, 819

Blanton, M. R., Lupton, R. H., Schlegel, D. J., et al. 2005, ApJ, 631, 208

Bos, E. G. P., van de Weygaert, R., Dolag, K., \& Pettorino, V. 2012, MNRAS, 426, 440

Bracewell, R. 1999, The Fourier Transform and its Applications, 3rd edn (New York: McGraw-Hill), 262

Brouwer, M. M., Demchenko, V., Harnois-Déraps, J., et al. 2018, MNRAS, 481, 5189

Cai, Y.-C., Cole, S., Jenkins, A., \& Frenk, C. S. 2010, MNRAS, 407, 201

Cai, Y.-C., Neyrinck, M. C., Szapudi, I., Cole, S., \& Frenk, C. S. 2014, ApJ, 786, 110

Cai, Y.-C., Padilla, N., \& Li, B. 2015, MNRAS, 451, 1036

Cai, Y.-C., Taylor, A., Peacock, J. A., \& Padilla, N. 2016, MNRAS, 462, 2465
Cai, Y.-C., Neyrinck, M., Mao, Q., et al. 2017, MNRAS, 466, 3364 Carretero, J., Castander, F. J., Gaztañaga, E., Crocce, M., \& Fosalba, P. 2015, MNRAS, 447, 646

Carretero, J., Tallada, P., Casals, J., et al. 2017, Proceedings of the European Physical Society Conference on High Energy Physics, 5-12 July, 488

Chan, K. C., \& Hamaus, N. 2021, Phys. Rev. D, 103, 043502

Chan, K. C., Hamaus, N., \& Desjacques, V. 2014, Phys. Rev. D, 90, 103521

Chan, K. C., Hamaus, N., \& Biagetti, M. 2019, Phys. Rev. D, 99, 121304

Chantavat, T., Sawangwit, U., \& Wandelt, B. D. 2017, ApJ, 836, 156

Clampitt, J., \& Jain, B. 2015, MNRAS, 454, 3357

Clampitt, J., Cai, Y.-C., \& Li, B. 2013, MNRAS, 431, 749

Contarini, S., Ronconi, T., Marulli, F., et al. 2019, MNRAS, 488, 3526

Contarini, S., Marulli, F., Moscardini, L., et al. 2021, MNRAS, 504, 5021

Correa, C. M., Paz, D. J., Padilla, N. D., et al. 2019, MNRAS, 485, 5761

Correa, C. M., Paz, D. J., Sánchez, A. G., et al. 2021, MNRAS, 500, 911

Correa, C. M., Paz, D. J., Padilla, N. D., et al. 2022, MNRAS, 509, 1871

Costille, A., Caillat, A., Rossin, C., et al. 2018, SPIE Conf. Ser., 10698, 106982B

Cousinou, M. C., Pisani, A., Tilquin, A., et al. 2019, Astron. Comput., 27, 53

Crocce, M., Castander, F. J., Gaztañaga, E., Fosalba, P., \& Carretero, J. 2015 MNRAS, 453, 1513

Cropper, M., Pottinger, S., Azzollini, R., et al. 2018, SPIE Conf. Ser., 10698, 1069828

Davies, C. T., Cautun, M., \& Li, B. 2019, MNRAS, 490, 4907

Dawson, K. S., Schlegel, D. J., Ahn, C. P., et al. 2013, AJ, 145, 10

Dawson, K. S., Kneib, J.-P., Percival, W. J., et al. 2016, AJ, 151, 44

de Jong, J. T. A., Verdoes Kleijn, G. A., Kuijken, K. H., \& Valentijn, E. A. 2013, Exp. Astron., 35, 25

Dolag, K., Komatsu, E., \& Sunyaev, R. 2016, MNRAS, 463, 1797

Eisenstein, D. J., Weinberg, D. H., Agol, E., et al. 2011, AJ, 142, 72

Euclid Collaboration (Blanchard, A., et al.) 2020, A\&A, 642, A191

Falck, B., Koyama, K., Zhao, G.-B., \& Cautun, M. 2018, MNRAS, 475, 3262

Fang, Y., Hamaus, N., Jain, B., et al. 2019, MNRAS, 490, 3573

Favole, G., Granett, B. R., Silva Lafaurie, J., \& Sapone, D. 2021, MNRAS, 505, 5833

Foreman-Mackey, D., Farr, W., Sinha, M., et al. 2019, J. Open Sour. Softw., 4, 1864

Forero-Sánchez, D., Zhao, C., Tao, C., et al. 2021, MNRAS, submitted [arXiv:2107.02950]

Gonçalves, R. S., Carvalho, G. C., Andrade, U., et al. 2021, JCAP, 2021, 029

Górski, K. M., Hivon, E., Banday, A. J., et al. 2005, ApJ, 622, 759

Granett, B. R., Neyrinck, M. C., \& Szapudi, I. 2008, ApJ, 683, L99

Grasshorn Gebhardt, H. S., Jeong, D., Awan, H., et al. 2019, ApJ, 876, 32

Gregory, S. A., \& Thompson, L. A. 1978, ApJ, 222, 784

Gruen, D., Friedrich, O., Amara, A., et al. 2016, MNRAS, 455, 3367

Guzzo, L., Scodeggio, M., Garilli, B., et al. 2014, A\&A, 566, A108

Habouzit, M., Pisani, A., Goulding, A., et al. 2020, MNRAS, 493, 899

Hahn, O., Porciani, C., Carollo, C. M., \& Dekel, A. 2007, MNRAS, 375, 489

Hahn, O., Angulo, R. E., \& Abel, T. 2015, MNRAS, 454, 3920

Hamaus, N., Wandelt, B. D., Sutter, P. M., Lavaux, G., \& Warren, M. S. 2014a, Phys. Rev. Lett., 112, 041304

Hamaus, N., Sutter, P. M., Lavaux, G., \& Wandelt, B. D. 2014b, JCAP, 12, 013 Hamaus, N., Sutter, P. M., \& Wandelt, B. D. 2014c, Phys. Rev. Lett., 112, 251302

Hamaus, N., Sutter, P. M., Lavaux, G., \& Wandelt, B. D. 2015, JCAP, 11, 036

Hamaus, N., Pisani, A., Sutter, P. M., et al. 2016, Phys. Rev. Lett., 117, 091302

Hamaus, N., Cousinou, M.-C., Pisani, A., et al. 2017, JCAP, 7, 014

Hamaus, N., Pisani, A., Choi, J.-A., et al. 2020, JCAP, 2020, 023

Harris, C. R., Millman, K. J., van der Walt, S. J., et al. 2020, Nature, 585, 357

Hartlap, J., Simon, P., \& Schneider, P. 2007, A\&A, 464, 399

Hawken, A. J., Granett, B. R., Iovino, A., et al. 2017, A\&A, 607, A54

Hawken, A. J., Aubert, M., Pisani, A., et al. 2020, JCAP, 2020, 012

Hickstein, D. D., Gibson, S. T., Yurchak, R., Das, D. D., \& Ryazanov, M. 2019, Rev. Sci. Instrum., 90, 065115

Hoyle, F., Rojas, R. R., Vogeley, M. S., \& Brinkmann, J. 2005, ApJ, 620, 618

Hunter, J. D. 2007, Comput. Sci. Eng., 9, 90

Ilić, S., Langer, M., \& Douspis, M. 2013, A\&A, 556, A51

Jõeveer, M., Einasto, J., \& Tago, E. 1978, MNRAS, 185, 357

Jackson, J. C. 1972, MNRAS, 156, 1P

Jennings, E., Li, Y., \& Hu, W. 2013, MNRAS, 434, 2167

Jones, D. H., Saunders, W., Colless, M., et al. 2004, MNRAS, 355, 747

Kaiser, N. 1987, MNRAS, 227, 1

Kirshner, R. P., Oemler, A., Jr., Schechter, P. L., \& Shectman, S. A. 1981, ApJ, 248, L57

Kitaura, F.-S., Chuang, C.-H., Liang, Y., et al. 2016, Phys. Rev. Lett., 116, 171301

Kovács, A., \& García-Bellido, J. 2016, MNRAS, 462, 1882

Kovács, A., Sánchez, C., García-Bellido, J., et al. 2019, MNRAS, 484, 5267

Kreckel, K., Platen, E., Aragón-Calvo, M. A., et al. 2012, AJ, 144, 16

Kreisch, C. D., Pisani, A., Carbone, C., et al. 2019, MNRAS, 488, 4413 
Kreisch, C. D., Pisani, A., Villaescusa-Navarro, F., et al. 2021, ApJ, submitted [arXiv:2107.02304]

Lahav, O., Lilje, P. B., Primack, J. R., \& Rees, M. J. 1991, MNRAS, 251, 128

Landy, S. D., \& Szalay, A. S. 1993, ApJ, 412, 64

Laureijs, R., Amiaux, J., Arduini, S., et al. 2011, ArXiv e-prints [arXiv:1110.3193]

Laurent, P., Le Goff, J.-M., Burtin, E., et al. 2016, JCAP, 2016, 060

Lavaux, G., \& Wandelt, B. D. 2012, ApJ, 754, 109

Leclercq, F., Jasche, J., Sutter, P. M., Hamaus, N., \& Wandelt, B. 2015, JCAP, 3 , 047

Lee, J., \& Park, D. 2009, ApJ, 696, L10

Leung, A. S., Acquaviva, V., Gawiser, E., et al. 2017, ApJ, 843, 130

Lewis, A. 2019, ArXiv e-prints [arXiv:1910.13970]

Lewis, A., Challinor, A., \& Lasenby, A. 2000, ApJ, 538, 473

Liang, Y., Zhao, C., Chuang, C.-H., Kitaura, F.-S., \& Tao, C. 2016, MNRAS, 459,4020

Linder, E. V. 2005, Phys. Rev. D, 72, 043529

Mao, Q., Berlind, A. A., Scherrer, R. J., et al. 2017, ApJ, 835, 160

Marulli, F., Bolzonella, M., Branchini, E., et al. 2013, A\&A, 557, A17

Marulli, F., Veropalumbo, A., Sereno, M., et al. 2018, A\&A, 620, A1

Massara, E., Villaescusa-Navarro, F., Viel, M., \& Sutter, P. M. 2015, JCAP, 11 018

Massara, E., Ho, S., Hirata, C. M., et al. 2021, MNRAS, 508, 4193

Melchior, P., Sutter, P. M., Sheldon, E. S., Krause, E., \& Wandelt, B. D. 2014, MNRAS, 440, 2922

Nadathur, S., \& Crittenden, R. 2016, ApJ, 830, L19

Nadathur, S., Carter, P. M., Percival, W. J., Winther, H. A., \& Bautista, J. E. 2019 Phys. Rev. D, 100, 023504

Nadathur, S., Woodfinden, A., Percival, W. J., et al. 2020, MNRAS, 499, 4140

Neyrinck, M. C. 2008, MNRAS, 386, 2101

Ntelis, P., Hamilton, J.-C., Le Goff, J.-M., et al. 2017, JCAP, 2017, 019

Paillas, E., Cautun, M., Li, B., et al. 2019, MNRAS, 484, 1149

Paillas, E., Cai, Y.-C., Padilla, N., \& Sánchez, A. G. 2021, MNRAS, 505, 5731

Pan, D. C., Vogeley, M. S., Hoyle, F., Choi, Y.-Y., \& Park, C. 2012, MNRAS, 421, 926

Panchal, R. R., Pisani, A., \& Spergel, D. N. 2020, ApJ, 901, 87

Patiri, S. G., Prada, F., Holtzman, J., Klypin, A., \& Betancort-Rijo, J. 2006 MNRAS, 372, 1710

Paz, D., Lares, M., Ceccarelli, L., Padilla, N., \& Lambas, D. G. 2013, MNRAS, 436, 3480

Peebles, P. J. E. 1980, The Large-Scale Structure of the Universe (Princeton: Princeton University Press)

Peebles, P. J. E. 2001, ApJ, 557, 495

Perico, E. L. D., Voivodic, R., Lima, M., \& Mota, D. F. 2019, A\&A, 632, A52

Pisani, A., Lavaux, G., Sutter, P. M., \& Wandelt, B. D. 2014, MNRAS, 443, 3238

Pisani, A., Sutter, P. M., Hamaus, N., et al. 2015a, Phys. Rev. D, 92, 083531

Pisani, A., Sutter, P. M., \& Wandelt, B. D. 2015b, ArXiv e-prints [arXiv:1506.07982]

Pisani, A., Massara, E., Spergel, D. N., et al. 2019, BAAS, 51, 40

Planck Collaboration XIII. 2016, A\&A, 594, A13

Planck Collaboration VI. 2020, A\&A, 641, A6

Platen, E., van de Weygaert, R., \& Jones, B. J. T. 2007, MNRAS, 380, 551

Pollina, G., Baldi, M., Marulli, F., \& Moscardini, L. 2016, MNRAS, 455 3075

Pollina, G., Hamaus, N., Dolag, K., et al. 2017, MNRAS, 469, 787

Pollina, G., Hamaus, N., Paech, K., et al. 2019, MNRAS, 487, 2836

Potter, D., Stadel, J., \& Teyssier, R. 2017, Comput. Astrophys. Cosmol., 4, 2

Pullen, A. R., Hirata, C. M., Doré, O., \& Raccanelli, A. 2016, PASJ, 68, 12

Reed, D. S., Schneider, A., Smith, R. E., et al. 2015, MNRAS, 451, 4413

Ricciardelli, E., Cava, A., Varela, J., \& Quilis, V. 2014, MNRAS, 445, 4045

Ronconi, T., Contarini, S., Marulli, F., Baldi, M., \& Moscardini, L. 2019 MNRAS, 488, 5075

Ryden, B. S. 1995, ApJ, 452, 25

Sahlén, M. 2019, Phys. Rev. D, 99, 063525

Sahlén, M., \& Silk, J. 2018, Phys. Rev. D, 97, 103504

Sánchez, A. G. 2020, Phys. Rev. D, 102, 123511

Sánchez, C., Clampitt, J., Kovacs, A., et al. 2017a, MNRAS, 465, 746

Sánchez, A. G., Scoccimarro, R., Crocce, M., et al. 2017b, MNRAS, 464 1640

Schaye, J., Crain, R. A., Bower, R. G., et al. 2015, MNRAS, 446, 521

Schuster, N., Hamaus, N., Pisani, A., et al. 2019, JCAP, 2019, 055

Scrimgeour, M. I., Davis, T., Blake, C., et al. 2012, MNRAS, 425, 116

Shandarin, S. F. 2011, JCAP, 5, 015

Sheth, R. K., \& van de Weygaert, R. 2004, MNRAS, 350, 517

Spolyar, D., Sahlén, M., \& Silk, J. 2013, Phys. Rev. Lett., 111, 241103

Springel, V. 2005, MNRAS, 364, 1105
Sutter, P. M., Lavaux, G., Wandelt, B. D., \& Weinberg, D. H. 2012a, ApJ, 761, 44

Sutter, P. M., Lavaux, G., Wandelt, B. D., \& Weinberg, D. H. 2012b, ApJ, 761, 187

Sutter, P. M., Pisani, A., Wandelt, B. D., \& Weinberg, D. H. 2014a, MNRAS, 443, 2983

Sutter, P. M., Elahi, P., Falck, B., et al. 2014b, MNRAS, 445, 1235

Sutter, P. M., Lavaux, G., Hamaus, N., et al. 2014c, MNRAS, 442, 462

Sutter, P. M., Lavaux, G., Hamaus, N., et al. 2015, Astron. Comput., 9, 1

Tallada, P., Carretero, J., Casals, J., et al. 2020, Astron. Comput., 32, 100391

The Dark Energy Survey Collaboration 2005, ArXiv e-prints [arXiv:astro-ph/0510346]

van de Weygaert, R., \& Schaap, W. 2009, The Cosmic Web: Geometric Analysis (Berlin: Springer), 665, 291

van de Weygaert, R., \& van Kampen, E. 1993, MNRAS, 263, 481

Verza, G., Pisani, A., Carbone, C., Hamaus, N., \& Guzzo, L. 2019, JCAP, 2019. 040

Virtanen, P., Gommers, R., Oliphant, T. E., et al. 2020, Nat. Meth., 17, 261

Voivodic, R., Lima, M., Llinares, C., \& Mota, D. F. 2017, Phys. Rev. D, 95, 024018

Voivodic, R., Rubira, H., \& Lima, M. 2020, JCAP, 2020, 033

White, S. D. M., Frenk, C. S., Davis, M., \& Efstathiou, G. 1987, ApJ, 313, 505

Wilson, C., \& Bean, R. 2021, Phys. Rev. D, 104, 023512

Yang, L. F., Neyrinck, M. C., Aragón-Calvo, M. A., Falck, B., \& Silk, J. 2015, MNRAS, 451, 3606

Zehavi, I., Zheng, Z., Weinberg, D. H., et al. 2011, ApJ, 736, 59

Zeldovich, Y. B. 1970, A\&A, 5, 84

Zhang, G., Li, Z., Liu, J., et al. 2020, Phys. Rev. D, 102, 083537

Zivick, P., Sutter, P. M., Wandelt, B. D., Li, B., \& Lam, T. Y. 2015, MNRAS, 451,4215

Zonca, A., Singer, L., Lenz, D., et al. 2019, J. Open Sour. Softw., 4, 1298

1 Universitäts-Sternwarte München, Fakultät für Physik, LudwigMaximilians-Universität München, Scheinerstrasse 1, 81679 München, Germany e-mail: n.hamaus@physik. lmu.de

2 University of Lyon, UCB Lyon 1, CNRS/IN2P3, IUF, IP2I, Lyon, France

3 Aix-Marseille Univ., CNRS/IN2P3, CPPM, Marseille, France

4 Department of Astrophysical Sciences, Peyton Hall, Princeton University, Princeton, NJ 08544, USA

5 Dipartimento di Fisica e Astronomia "Augusto Righi" - Alma Mater Studiorum Universitá di Bologna, Viale Berti Pichat 6/2, 40127 Bologna, Italy

6 INFN-Sezione di Bologna, Viale Berti Pichat 6/2, 40127 Bologna, Italy

7 INAF-IASF Bologna, Via Piero Gobetti 101, 40129 Bologna, Italy

8 INFN-Padova, Via Marzolo 8, 35131 Padova, Italy

9 Dipartimento di Fisica e Astronomia "G. Galilei", Universitá di Padova, Via Marzolo 8, 35131 Padova, Italy

10 Sorbonne Universités, UPMC Univ. Paris 6 et CNRS, UMR 7095, Institut d'Astrophysique de Paris, 98bis bd Arago, 75014 Paris, France

11 Max Planck Institute for Extraterrestrial Physics, Giessenbachstr. 1, 85748 Garching, Germany

12 INFN-Sezione di Genova, Via Dodecaneso 33, 16146 Genova, Italy

13 Dipartimento di Fisica, Universitá degli Studi di Genova, and INFNSezione di Genova, Via Dodecaneso 33, 16146 Genova, Italy

14 INFN-Sezione di Milano, Via Celoria 16, 20133 Milano, Italy

15 INAF-IASF Milano, Via Alfonso Corti 12, 20133 Milano, Italy

16 Dipartimento di Fisica “Aldo Pontremoli”, Universitá degli Studi di Milano, Via Celoria 16, 20133 Milano, Italy

17 INAF-Osservatorio Astronomico di Brera, Via Brera 28, 20122 Milano, Italy

18 Universidad de la Laguna, 38206 San Cristóbal de La Laguna, Tenerife, Spain

19 Instituto de Astrofísica de Canarias, Calle Vía Làctea s/n, 38204 San Cristòbal de la Laguna, Tenerife, Spain

20 Dipartimento di Fisica e Astronomia “Augusto Righi" - Alma Mater Studiorum Universitá di Bologna, Via Piero Gobetti 93/2, 40129 Bologna, Italy 
${ }^{21}$ INAF-Osservatorio di Astrofisica e Scienza dello Spazio di Bologna, Via Piero Gobetti 93/3, 40129 Bologna, Italy

22 Department of Physics and Astronomy, University of Waterloo, Waterloo, Ontario N2L 3G1, Canada

${ }^{23}$ Centre for Astrophysics, University of Waterloo, Waterloo, Ontario N2L 3G1, Canada

${ }^{24}$ Perimeter Institute for Theoretical Physics, Waterloo, Ontario N2L 2Y5, Canada

25 Institute of Theoretical Astrophysics, University of Oslo, PO Box 1029, Blindern 0315, Oslo, Norway

26 Swedish Collegium for Advanced Study, Thunbergsvägen 2, 75238 Uppsala, Sweden

27 Theoretical Astrophysics, Department of Physics and Astronomy, Uppsala University, Box 515, 75120 Uppsala, Sweden

28 Université St Joseph, UR EGFEM, Faculty of Sciences, Beirut, Lebanon

29 Institut de Recherche en Astrophysique et Planétologie (IRAP), Université de Toulouse, CNRS, UPS, CNES, 14 Av. Edouard Belin, 31400 Toulouse, France

${ }^{30}$ Mullard Space Science Laboratory, University College London, Holmbury St Mary, Dorking, Surrey RH5 6NT, UK

31 INAF-Osservatorio Astrofisico di Torino, Via Osservatorio 20, 10025 Pino Torinese, TO, Italy

32 INFN-Sezione di Roma Tre, Via della Vasca Navale 84, 00146 Roma, Italy

33 Department of Mathematics and Physics, Roma Tre University, Via della Vasca Navale 84, 00146 Rome, Italy

34 INAF-Osservatorio Astronomico di Capodimonte, Via Moiariello 16, 80131 Napoli, Italy

35 Instituto de Astrofísica e Ciências do Espaço, Universidade do Porto, CAUP, Rua das Estrelas, 4150-762 Porto, Portugal

36 Port d'Informació Científica, Campus UAB, C. Albareda s/n, 08193 Bellaterra, Barcelona, Spain

37 Institut de Física d'Altes Energies (IFAE), The Barcelona Institute of Science and Technology, Campus UAB, 08193 Bellaterra, Barcelona, Spain

38 Institute of Space Sciences (ICE, CSIC), Campus UAB, Carrer de Can Magrans, s/n, 08193 Barcelona, Spain

39 Institut d'Estudis Espacials de Catalunya (IEEC), Carrer Gran Capitá 2-4, 08034 Barcelona, Spain

40 INAF-Osservatorio Astronomico di Roma, Via Frascati 33, 00078 Monteporzio Catone, Italy

41 Department of Physics "E. Pancini”, University Federico II, Via Cinthia 6, 80126 Napoli, Italy

42 INFN Section of Naples, Via Cinthia 6, 80126 Napoli, Italy

43 INAF-Osservatorio Astrofisico di Arcetri, Largo E. Fermi 5, 50125 Firenze, Italy

44 Institut National de Physique Nucléaire et de Physique des Particules, 3 rue Michel-Ange, 75794 Paris Cedex 16, France

45 Centre National d'Etudes Spatiales, Toulouse, France

46 Institute for Astronomy, University of Edinburgh, Royal Observatory, Blackford Hill, Edinburgh EH9 3HJ, UK

47 European Space Agency/ESRIN, Largo Galileo Galilei 1, 00044 Frascati, Roma, Italy

48 ESAC/ESA, Camino Bajo del Castillo, s/n., Urb. Villafranca del Castillo, 28692 Villanueva de la Cañada, Madrid, Spain

49 Univ. Lyon, Univ. Claude Bernard Lyon 1, CNRS/IN2P3, IP2I Lyon, UMR 5822, 69622 Villeurbanne, France

50 Departamento de Física, Faculdade de Ciências, Universidade de Lisboa, Edifício C8, Campo Grande, 1749-016 Lisboa, Portugal

51 Instituto de Astrofísica e Ciências do Espaço, Faculdade de Ciências, Universidade de Lisboa, Campo Grande, 1749-016 Lisboa, Portugal

52 Department of Astronomy, University of Geneva, ch. d'Ecogia 16, 1290 Versoix, Switzerland

53 Université Paris-Saclay, CNRS, Institut d'Astrophysique Spatiale, 91405 Orsay, France
54 Department of Physics, Oxford University, Keble Road, Oxford OX1 3RH, UK

55 INAF-Osservatorio Astronomico di Trieste, Via G. B. Tiepolo 11, 34131 Trieste, Italy

56 Istituto Nazionale di Astrofisica (INAF) - Osservatorio di Astrofisica e Scienza dello Spazio (OAS), Via Gobetti 93/3, 40127 Bologna, Italy

57 Istituto Nazionale di Fisica Nucleare, Sezione di Bologna, Via Irnerio 46, 40126 Bologna, Italy

58 INAF-Osservatorio Astronomico di Padova, Via dell'Osservatorio 5, 35122 Padova, Italy

59 Jet Propulsion Laboratory, California Institute of Technology, 4800 Oak Grove Drive, Pasadena, CA 91109, USA

${ }^{60}$ von Hoerner \& Sulger GmbH, SchloßPlatz 8, 68723 Schwetzingen, Germany

61 Max-Planck-Institut für Astronomie, Königstuhl 17, 69117 Heidelberg, Germany

62 AIM, CEA, CNRS, Université Paris-Saclay, Université de Paris, 91191 Gif-sur-Yvette, France

63 Université de Genève, Département de Physique Théorique and Centre for Astroparticle Physics, 24 quai Ernest-Ansermet, 1211 Genève 4 , Switzerland

64 Department of Physics and Helsinki Institute of Physics, University of Helsinki, Gustaf Hällströmin katu 2, 00014 Helsinki, Finland

65 NOVA Optical Infrared Instrumentation Group at ASTRON, Oude Hoogeveensedijk 4, 7991 PD Dwingeloo, The Netherlands

66 Argelander-Institut für Astronomie, Universität Bonn, Auf dem Hügel 71, 53121 Bonn, Germany

67 Institute for Computational Cosmology, Department of Physics, Durham University, South Road, Durham DH1 3LE, UK

68 Université Côte d'Azur, Observatoire de la Côte d'Azur, CNRS, Laboratoire Lagrange, Bd de l'Observatoire, CS 34229, 06304 Nice Cedex 4, France

69 University of Applied Sciences and Arts of Northwestern Switzerland, School of Engineering, 5210 Windisch, Switzerland

70 California Institute of Technology, 1200 E California Blvd, Pasadena, CA 91125, USA

71 Institute of Physics, Laboratory of Astrophysics, Ecole Polytechnique Fédérale de Lausanne (EPFL), Observatoire de Sauverny, 1290 Versoix, Switzerland

72 European Space Agency/ESTEC, Keplerlaan 1, 2201 AZ Noordwijk, The Netherlands

73 Department of Physics and Astronomy, University of Aarhus, Ny Munkegade 120, 8000 Aarhus C, Denmark

${ }^{74}$ Institute of Space Science, Bucharest 077125, Romania

75 Departamento de Astrofísica, Universidad de La Laguna, $38206 \mathrm{La}$ Laguna, Tenerife, Spain

76 Centro de Investigaciones Energéticas, Medioambientales y Tecnológicas (CIEMAT), Avenida Complutense 40, 28040 Madrid, Spain

77 Instituto de Astrofísica e Ciências do Espaço, Faculdade de Ciências, Universidade de Lisboa, Tapada da Ajuda 1349-018, Lisboa, Portugal

78 Universidad Politécnica de Cartagena, Departamento de Electrónica y Tecnología de Computadoras, 30202 Cartagena, Spain

79 Kapteyn Astronomical Institute, University of Groningen, PO Box 800, 9700 AV Groningen, The Netherlands

80 Infrared Processing and Analysis Center, California Institute of Technology, Pasadena, CA 91125, USA

81 Dipartimento di Fisica e Astronomia, Universitá di Bologna, Via Gobetti 93/2, 40129 Bologna, Italy

82 INFN-Sezione di Torino, Via P. Giuria 1, 10125 Torino, Italy

83 Dipartimento di Fisica, Universitá degli Studi di Torino, Via P. Giuria 1, 10125 Torino, Italy

${ }^{84}$ Université de Paris, CNRS, Astroparticule et Cosmologie, 75013 Paris, France 